\title{
Magnetic field spectrum at cosmological recombination revisited
}

\author{
Shohei Saga, ${ }^{1}$ Kiyotomo Ichiki, ${ }^{1,2}$ Keitaro Takahashi, ${ }^{3}$ and Naoshi Sugiyama ${ }^{1,2,4}$ \\ ${ }^{1}$ Department of Physics and Astrophysics, Nagoya University, Aichi 464-8602, Japan $*$ \\ ${ }^{2}$ Kobayashi-Maskawa Institute for the Origin of Particles and the Universe, Nagoya University, Aichi 464-8602, Japan \\ ${ }^{3}$ University of Kumamoto, 2-39-1, Kurokami, Kumamoto 860-8555, Japan \\ ${ }^{4}$ Kavli Institute for the Physics and Mathematics of the Universe (Kavli IPMU), \\ The University of Tokyo, Chiba 277-8582, Japan
}

\begin{abstract}
If vector type perturbations are present in the primordial plasma before recombination, the generation of magnetic fields is known to be inevitable through the Harrison mechanism. In the context of the standard cosmological perturbation theory, nonlinear couplings of first-order scalar perturbations create second-order vector perturbations, which generate magnetic fields. Here we reinvestigate the generation of magnetic fields at second-order in cosmological perturbations on the basis of our previous study, and extend it by newly taking into account the time evolution of purely second-order vector perturbations with a newly developed second-order Boltzmann code. We confirm that the amplitude of magnetic fields from the product-terms of the first-order scalar modes is consistent with the result in our previous study. However, we find, both numerically and analytically, that the magnetic fields from the purely second-order vector perturbations partially cancel out the magnetic fields from one of the product-terms of the first-order scalar modes, in the tight coupling regime in the radiation dominated era. Therefore, the amplitude of the magnetic fields on small scales, $k \gtrsim 10 \mathrm{hMpc}^{-1}$, is smaller than the previous estimates. The amplitude of the generated magnetic fields at cosmological recombination is about $B_{\text {rec }}=5.0 \times 10^{-24}$ Gauss on $k=5.0 \times 10^{-1} h_{\mathrm{Mpc}^{-1}}$. Finally, we discuss the reason for the discrepancies that exist in estimates of the amplitude of magnetic fields among other authors.
\end{abstract}

\section{INTRODUCTION}

The presence of magnetic fields on large scales is established by current observations [1 [5]. Such cosmological magnetic fields coevolve with the Universe, e.g., astrophysical objects, cosmic microwave background radiation (CMB), large scale structure, and inflation. Recent observations indicate that cosmological magnetic fields have the strength about micro-Gauss on Mpc scales (see, e.g., Refs. [1, 2, 5, [8], and references therein). Moreover, the pair-echo method $\left[9\right.$ 13] determines the lower bound of strength in the intergalactic magnetic fields as $B \gtrsim O\left(10^{-22}\right)$ Gauss. The remarkable progress of observations indicates that cosmological magnetic fields appear everywhere even in the cosmic voids. Furthermore, the future experiments of the radio telescope such as Square Kilometer Array can survey much deeper and wider regions, and give us rich information about coevolution between cosmological magnetic fields and baryonic matters. However, very interestingly, the origin of cosmological magnetic fields is not entirely revealed.

The key process related to evolution of magnetic fields is the dynamo mechanism [7, 14 16], which is the amplification mechanism of magnetic fields in the nonlinear magnetohydrodynamics. In the stars, galaxies, and galaxy clusters, their nonlinear evolution can amplify seed magnetic fields, and the strength of seed fields is about $10^{-20} \sim 10^{-30}$ Gauss [17]. Generally, the dynamo mechanism cannot generate magnetic fields from the absence of seed fields but amplify the seed fields, which should be set before the dynamo mechanism works. Therefore, when we believe that the origin of cosmological magnetic fields is as a result of amplification of seed fields by the dynamo mechanism, seed fields must be created in the early stage of the universe, namely before cosmological recombination.

One of the candidates to create seed fields is the quantum fluctuations of the electromagnetic fields in the inflation era. During the inflation era, the scale of fluctuations is extended beyond the Hubble horizon due to the nearly exponential expansion. At first glance, it is possible to rely on this scenario to generate large-scale magnetic fields. However, this scenario does not work since the standard Maxwell theory has the symmetry under the conformal transformation. Under this symmetry, the vector fields, such as magnetic fields, undergo decaying only and become negligible. In other words, when the conformal invariance is broken, inflationary magnetogenesis possibly works. In many of the previous studies [18 22], the authors have introduced interaction between the electromagnetic fields and the dilaton-like scalar field to break conformal invariance. However, even in this case, there are other problems in the inflationary magnetogenesis, i.e., strong-coupling and backreaction problems [23, 24]. These problems make the

*Electronic address: saga.shohei@nagoya-u.jp 
situation worse. Therefore we can conclude that it is difficult to generate seed fields during the inflation era alone. In the more recent studies 25 27], a new interaction between electromagnetic fields and axion-like pseudoscalar field is added in the context of dilaton-like magnetogenesis. This interaction ends up with generation of helical magnetic fields on small scales. The inverse cascade can transfer helical magnetic fields from smaller scales to much larger scales owing to conservation of the magnetic helicity. We therefore expect the existence of magnetic fields on all scales in later epochs although the detailed numerical estimation is needed [28, 29].

The cosmological phase transition is another possibility to generate seed fields (e.g., Refs. [7, 8]). In general, phase transitions release the free energy and electric charges. The released free energy is converted into the electric currents. If these electric currents have rotational components, cosmological seed fields are induced at epochs of phase transitions. However, the coherent length of seed fields generated in the cosmological phase transitions cannot exceed the Hubble horizon scale at that time due to the causality. Therefore phase transitions alone are not able to explain observed large-scale magnetic fields [30].

Another category of generation mechanism is originated from astrophysical phenomena. For example, the Biermann battery is one of the candidates of generation mechanism after recombination. The gravitational force can be described as a gradient of the scalar potential and hence cannot generate vorticity. However, the Biermann battery, which is nonadiabatic phenomena such as shocks, can generate vorticity and subsequently, seed fields are induced with the amplitude about $10^{-17}$ Gauss in protogalaxies [31], $10^{-17} \sim 10^{-14}$ Gauss in supernova remnants [32], and $\sim 10^{-21}$ Gauss in galaxies [33]. The Weibel instability, which is microscopic instability in the plasma, can amplify tiny seed fields at the epoch of structure formation [34, 35]. When the velocity distribution of plasma particles has an anisotropy in the phase space, the isotropized process of the velocity distribution releases the energy, and subsequently, the energy is converted into magnetic fields. Accordingly, magnetic fields amplified by the Weibel instability have a quite large amplitude of about $10^{-7}$ Gauss [34, 35]. However, the Biermann battery and Weibel instability can only work with the existence of baryonic matters or astrophysical objects. Therefore it is difficult to explain the origin of intergalactic magnetic fields or magnetic fields in the voids.

Yet another interesting mechanism of generating magnetic fields is the Harrison mechanism [36] in which magnetic fields are generated via vorticity of the primordial plasma. In Ref. [37], the authors have formulated the Harrison mechanism based on the cosmological perturbation theory in the primordial plasma which is a multicomponent system composed of photons, electrons, protons, dark matters, and neutrinos. In this system, photons and electrons or protons interact with each other through the Compton scattering. However, photons push electrons more frequently than protons because of the difference of scattering rates. Accordingly, the charge separation takes place. If there exist rotation-type electric fields, magnetic fields are generated. However, in the linear perturbation theory, the Harrison mechanism does not work because there is no growing mode solution for the vector-mode perturbations which induce rotation-type electric fields. In other words, the models including the active vector mode supplied by external sources, i.e., free-streaming neutrinos [38 40], cosmic defects [41, 42], and modified gravity with vector fields [43], can generate magnetic fields via the Harrison mechanism.

Moreover, it turns out that even standard cosmological perturbations can generate magnetic fields if we take into account contributions from higher-order perturbations. In fact, it is known that the second-order perturbation theory has not only the scalar mode but also the vector and tensor modes through the product of the first-order scalar perturbations. Recently, the second-order cosmological perturbation theory is well established in the context of the CMB formalisms [44 55]. For example, the B-mode polarization is calculated based on the second-order perturbation theory while there is no B-mode polarization in linear scalar perturbations.

Recently, generation of magnetic fields via the second-order perturbation has been studied in detail [56 60]. In these studies, the tight-coupling approximation is employed to estimate the amplitude of magnetic fields analytically. Each study has shown that the amplitude of generated magnetic fields is about $10^{-30} \sim 10^{-27}$ Gauss at recombination on Mpc scales. However, it is difficult to know the detail of the magnetic power spectrum since the tight-coupling approximation breaks down inside the horizon scale at recombination. By solving perturbation equations up to the second order without employing the tight-coupling approximation, it is possible to analyze the power spectrum of magnetic fields. In Ref. [61], the authors have evaluated the spectrum generated by the vorticity of charged particles, which are induced by the nonlinear coupling between the first-order density perturbations. And they have found that resultant comoving magnetic fields have the amplitude of about $10^{-29}$ Gauss at recombination on Mpc scales. Subsequently, in Refs. 62, 63], the authors have studied the Harrison mechanism including the anisotropic stress of photons. They have found that the amplitude of magnetic fields has $10^{-20}$ Gauss at recombination on Mpc scales. However, they ignore the purely second-order velocity difference between charged particles and photons in their analysis. In Ref. [64], the authors include the purely second-order effects for the first time and analyze the spectrum of magnetic fields on superhorizon scales. In these studies, however, there are some discrepancies which have to be clarified.

In this paper we numerically solve the vector mode of cosmological Einstein-Boltzmann equations at the second order including all the effects relevant to the generation of magnetic fields, with a newly developed numerical code. In 
addition we present analytic interpretations of the shapes and time evolutions of the power spectrum of magnetic fields on sub- and superhorizon scales, and make it clear what has caused the discrepancies among the previous studies. The paper is organized as follows. In Sec. II, we review the generation mechanism of magnetic fields. We decompose the evolution equation of magnetic fields up to the second order in terms of scalar and vector perturbations. It is shown that the magnetic fields are generated by the vector mode only. In Sec. III, we describe the perturbed Einstein-Boltzmann system up to the second order to compute the purely second-order perturbations, and show the tight-coupling solutions of the system. Time evolutions of magnetic fields and spectra are numerically evaluated and the analytic expressions are given in Sec. IV. We devote Sec. V to our discussions and conclusions.

Throughout this paper, we use the units in which $c=\hbar=1$ and the metric signature as $(-,+,+,+)$. We obey the rule that the subscripts and superscripts of the Greek characters and alphabets run from 0 to 3 and from 1 to 3 , respectively.

\section{GENERATION OF MAGNETIC FIELDS}

In this section, we review basic equations for the generation of magnetic fields [37, 65], i.e., perturbation equations of photon, proton, and electron fluids. While protons and electrons are conventionally treated as a single fluid, however, it is necessary to deal with proton and electron fluids separately in order to discuss the generation of magnetic fields. Let us begin with the Euler equations. Those are given by

$$
\begin{aligned}
& m_{\mathrm{p}} n u_{\mathrm{p}}^{\mu} u_{\mathrm{p} i ; \mu}-e n u_{\mathrm{p}}^{\mu} F_{i \mu}=C_{i}^{\mathrm{pe}}+C_{i}^{\mathrm{p} \gamma}, \\
& m_{\mathrm{e}} n u_{\mathrm{e}}^{\mu} u_{\mathrm{e} i ; \mu}+e n u_{\mathrm{e}}^{\mu} F_{i \mu}=C_{i}^{\mathrm{ep}}+C_{i}^{\mathrm{e} \gamma},
\end{aligned}
$$

where $m_{\mathrm{p}(\mathrm{e})}$ is the proton (electron) mass, $u_{\mathrm{p}(\mathrm{e})}$ is the bulk velocity of protons (electrons), $F_{\mu i}$ is the usual Maxwell tensor. The thermal pressures of proton and electron fluids are neglected. The right-hand side of Eqs. (11) and (2) represent the collision terms. The first terms in Eqs. (11) and (2) are collision terms for the Coulomb scattering between protons and electrons, which are given by [56]

$$
C_{i}^{\mathrm{pe}}=-C_{i}^{\mathrm{ep}}=-\left(u_{i}-u_{\mathrm{e} i}\right) e^{2} n^{2} \eta_{\mathrm{r}}
$$

where

$$
\eta_{\mathrm{r}}=\frac{\pi e^{2} m_{\mathrm{e}}^{1 / 2}}{\left(k_{B} T_{\mathrm{e}}\right)^{3 / 2}} \ln \Lambda \sim 9.4 \times 10^{-16} \sec \left(\frac{1+z}{10^{5}}\right)^{-3 / 2}\left(\frac{\ln \Lambda}{10}\right),
$$

is the resistivity of the plasma and $\ln \Lambda \sim \mathcal{O}(1)$ is the Coulomb logarithm, which is the almost constant parameter. As is well known, this term acts as the diffusion term in the evolution equation of magnetic field. The importance of the diffusion effect can be estimated by the diffusion scale,

$$
\lambda_{\text {diff }} \equiv \sqrt{\eta_{\mathrm{r}} \tau} \sim 100\left(\frac{\tau}{H_{0}^{-1}}\right)^{1 / 2} \mathrm{AU},
$$

above which magnetic field cannot diffuse in the time-scale $\tau$. Here $H_{0}=100 \mathrm{~h} \mathrm{~km} / \mathrm{s} / \mathrm{Mpc}$ is the present Hubble parameter with $h$ being the normalized Hubble parameter. Thus, at cosmological scales considered in this paper, this term can be safely neglected.

The other terms expressed by $C_{i}^{\mathrm{p}(\mathrm{e}) \gamma}$ are the collision terms for Compton scattering of protons (electrons) with photons. Since photons scatter off electrons preferentially compared with protons by a factor of $\left(m_{\mathrm{e}} / m_{\mathrm{p}}\right)^{2}$, we can safely drop the term $C_{i}^{\mathrm{p} \gamma}$ from the Euler equation of protons. This difference in collision terms between protons and electrons ensures that small difference in velocity between protons and electrons, that is, electric current, is indeed generated once the Compton scattering becomes effective. In the next subsection, we derive the explicit form of the Compton scattering term.

\section{A. Compton collision term}

Let us now evaluate the Compton scattering term. In the limit of completely elastic collisions between photons and electrons, this term vanishes. Typically, in the regime of interest in this paper, very little energy is transferred between electrons and photons in Compton scatterings. Therefore, it is a good approximation to expand the collision term systematically in powers of the energy transfer. 
Let us demonstrate this specifically. We consider the collision process

$$
\gamma\left(p^{\mu}\right)+e^{-}\left(q^{\mu}\right) \rightarrow \gamma\left(p^{\prime \mu}\right)+e^{-}\left(q^{\prime \mu}\right)
$$

where the quantities in the parentheses denote the particle momenta. To calculate this process, we evaluate the collision term in the Boltzmann equation of photons:

$$
\begin{aligned}
C[f]= & \frac{a}{E(p)} \int \frac{d^{3} p^{\prime}}{(2 \pi)^{3} 2 E\left(p^{\prime}\right)} \frac{d^{3} q}{(2 \pi)^{3} 2 E_{\mathrm{e}}(q)} \frac{d^{3} q^{\prime}}{(2 \pi)^{3} 2 E_{\mathrm{e}}\left(q^{\prime}\right)}|\mathcal{M}|^{2} \\
& \times(2 \pi)^{4} \delta^{4}\left(q^{\mu}+p^{\mu}-q^{\prime \mu}-p^{\prime \mu}\right)\left[g_{\mathrm{e}}\left(\boldsymbol{q}^{\prime}\right) f\left(\boldsymbol{p}^{\prime}\right)(1+f(\boldsymbol{p}))-g_{\mathrm{e}}(\boldsymbol{q}) f(\boldsymbol{p})\left(1+f\left(\boldsymbol{p}^{\prime}\right)\right)\right]
\end{aligned}
$$

where $f(\boldsymbol{p})$ and $g_{\mathrm{e}}(\boldsymbol{q})$ are the distribution functions of photons and electrons, $E_{\mathrm{e}}(q)=\sqrt{q^{2}+m_{\mathrm{e}}^{2}}$ is the energy of an electron, and the delta functions enforce the energy and momentum conservations. And $|\mathcal{M}|^{2}$ is the scattering amplitude for Compton scattering. We have dropped the Pauli blocking factor $\left(1-g_{\mathrm{e}}\right)$. The Pauli blocking factor can be always omitted safely in the epoch of interest, because $g_{\mathrm{e}}$ is very small after electron-positron annihilations.

Integrating over $q^{\prime}$, we obtain

$$
\begin{aligned}
C[f]= & \frac{a}{p} \int \frac{d^{3} p^{\prime}}{(2 \pi)^{3} 2 p^{\prime}} \frac{d^{3} q}{(2 \pi)^{3} 2 E_{\mathrm{e}}(q)} \frac{2 \pi}{2 E_{\mathrm{e}}\left(\left|\boldsymbol{q}+\boldsymbol{p}-\boldsymbol{p}^{\prime}\right|\right)}|\mathcal{M}|^{2} \\
& \times \delta\left[p-p^{\prime}+E_{\mathrm{e}}(q)-E_{\mathrm{e}}\left(\left|\boldsymbol{q}+\boldsymbol{p}-\boldsymbol{p}^{\prime}\right|\right)\right]\left[g_{\mathrm{e}}\left(\boldsymbol{q}+\boldsymbol{p}-\boldsymbol{p}^{\prime}\right) f\left(\boldsymbol{p}^{\prime}\right)(1+f(\boldsymbol{p}))-g_{\mathrm{e}}(\boldsymbol{q}) f(\boldsymbol{p})\left(1+f\left(\boldsymbol{p}^{\prime}\right)\right)\right] .
\end{aligned}
$$

In the regime of our interest, energy transfer through the Compton scattering is small and can be ignored in the first order density perturbations. As we already discussed earlier, however, it is essential to take the second-order couplings in the Compton scattering term into consideration for the generation of magnetic fields. Therefore, we expand the collision term up to the first order in powers of the energy transfer ${ }^{1}$, and keep terms up to the second order in density perturbations.

The expansion parameter is the energy transfer,

$$
E_{\mathrm{e}}(q)-E_{\mathrm{e}}\left(\left|\boldsymbol{q}+\boldsymbol{p}-\boldsymbol{p}^{\prime}\right|\right) \simeq \frac{\left(\boldsymbol{p}^{\prime}-\boldsymbol{p}\right) \cdot \boldsymbol{q}}{m_{\mathrm{e}}}-\frac{\left(\boldsymbol{p}-\boldsymbol{p}^{\prime}\right)}{2 m_{\mathrm{e}}},
$$

over the temperature of the universe. Employing $p \sim T$, we can estimate the order of this expansion parameter as $\mathcal{O}\left(\frac{p q}{m_{\mathrm{e}} T}\right) \sim \mathcal{O}\left(\frac{q}{m_{\mathrm{e}}}\right)$, which is small when electrons are nonrelativistic. Note that, in the cosmological Thomson regime, electrons in the thermal bath of photons are nonrelativistic, $p \sim \frac{q^{2}}{2 m_{\mathrm{e}}}$, and the energy of photons is much smaller than the rest mass of a electron, $p \ll m_{e}$. Thus, it also holds that $q \sim \sqrt{2 m_{\mathrm{e}} p} \gg p$, and the second term in Eq. (9) is usually smaller than the first one.

Now let us divide the collision integral into four parts, i.e., the denominators of the Lorentz invariant volume, the scattering amplitude, the delta function, and the distribution functions, and expand them due to the expansion parameter defined above. First of all, the denominator in the Lorentz invariant volume can be expanded to

$$
\begin{aligned}
\frac{1}{E_{\mathrm{e}}(q) E_{\mathrm{e}}\left(\left|\boldsymbol{q}+\boldsymbol{p}-\boldsymbol{p}^{\prime}\right|\right)} & =\left(m_{\mathrm{e}}+\frac{1}{2 m_{\mathrm{e}}} q^{2}\right)^{-1}\left(m_{\mathrm{e}}+\frac{1}{2 m_{\mathrm{e}}}\left|\boldsymbol{q}+\boldsymbol{p}-\boldsymbol{p}^{\prime}\right|\right)^{-1} \\
& \approx \frac{1}{m_{\mathrm{e}}^{2}}\left[1-\mathcal{E}_{\left(\frac{q}{m_{\mathrm{e}}}\right)^{2}}-\mathcal{E}_{\left(\frac{p q}{m_{\mathrm{e}}}\right)}-\mathcal{E}_{\left(\frac{p}{m_{\mathrm{e}}}\right)^{2}}\right]
\end{aligned}
$$

where

$$
\mathcal{E}_{\left(\frac{q}{m_{\mathrm{e}}}\right)^{2}}=\frac{q^{2}}{m_{\mathrm{e}}^{2}}, \quad \mathcal{E}_{\left(\frac{p q}{m_{\mathrm{e}}^{2}}\right)}=\frac{\left(\boldsymbol{p}-\boldsymbol{p}^{\prime}\right) \cdot \boldsymbol{q}}{m_{\mathrm{e}}^{2}}, \quad \mathcal{E}_{\left(\frac{p}{m_{\mathrm{e}}}\right)^{2}}=\frac{\left(\boldsymbol{p}-\boldsymbol{p}^{\prime}\right)^{2}}{2 m_{\mathrm{e}}^{2}} .
$$

Second, we consider the scattering amplitude. Fortunately, it has been known that the leading term (zeroth order term), obtained by multiplying together the first term in the delta function and the zeroth-order distribution functions, is zero. It means that we only have to keep up to the first order terms when we expand the scattering amplitude and

\footnotetext{
${ }^{1}$ However, we shall keep up to the second-order terms for the purpose of deriving the Einstein-Boltzmann system in section
} 
the energies, in order to keep the collision term up to the second order [66]. The scattering amplitude for Compton scattering in the rest frame of the electron is given by,

$$
\begin{aligned}
|\mathcal{M}|^{2} & =6 \pi m_{\mathrm{e}}^{2} \sigma_{T}\left[\frac{\tilde{p^{\prime}}}{\tilde{p}}+\frac{\tilde{p}}{\tilde{p}^{\prime}}-\sin ^{2} \tilde{\beta}\right], \\
\cos \tilde{\beta} & =\tilde{\hat{\boldsymbol{p}}} \cdot \tilde{\hat{\boldsymbol{p}}}^{\prime}
\end{aligned}
$$

where $\tilde{p}$ and $\tilde{p^{\prime}}$ are the energies of incident and scattered photons, $\tilde{\hat{\boldsymbol{p}}}$ and $\tilde{\hat{\boldsymbol{p}}}^{\prime}$ are the unit vectors of $\tilde{\boldsymbol{p}}$ and $\tilde{\boldsymbol{p}^{\prime}}$, respectively, denoting the directions of the photons in this frame. The Lorentz transformation with electron's velocity $\left(q / m_{\mathrm{e}}\right)$ gives the following relations,

$$
\begin{aligned}
\frac{p}{\tilde{p}} & =\frac{\sqrt{1-\left(q / m_{\mathrm{e}}\right)^{2}}}{1-\boldsymbol{p} \cdot \boldsymbol{q} /\left(p m_{\mathrm{e}}\right)} \\
p^{\mu} p_{\mu} & =\tilde{p}^{\mu} \tilde{p}_{\mu} .
\end{aligned}
$$

Using these relations, we evaluate the scattering amplitude in the CMB frame as 67

$$
|\mathcal{M}|^{2}=6 \pi m_{\mathrm{e}}^{2} \sigma_{T}\left[\mathcal{M}_{0}+\mathcal{M}_{\left(\frac{q}{m_{\mathrm{e}}}\right)}\right]
$$

where

$$
\mathcal{M}_{0}=1+\cos ^{2} \beta, \quad \mathcal{M}_{\left(\frac{q}{m_{\mathrm{e}}}\right)}=-2 \cos \beta(1-\cos \beta)\left[\frac{\boldsymbol{q}}{m_{\mathrm{e}}} \cdot\left(\hat{\boldsymbol{n}}+\hat{\boldsymbol{n}}^{\prime}\right)\right] .
$$

Here $\hat{\boldsymbol{n}}$ and $\hat{\boldsymbol{n}}^{\prime}$ are the unit vectors of $\boldsymbol{p}$ and $\boldsymbol{p}^{\prime}$, respectively.

Third, we expand the delta function to

$$
\begin{aligned}
\delta\left[p-p^{\prime}+E_{\mathrm{e}}(q)-E_{\mathrm{e}}\left(q^{\prime}\right)\right] \approx & \delta\left(p-p^{\prime}\right)+\left.\frac{\partial \delta\left[p-p^{\prime}+E e(q)-E_{\mathrm{e}}\left(q^{\prime}\right)\right]}{\partial p}\right|_{q=q^{\prime}}\left(E_{\mathrm{e}}(q)-E_{\mathrm{e}}\left(q^{\prime}\right)\right) \\
& +\left.\frac{1}{2} \frac{\partial^{2} \delta\left[p-p^{\prime}+E_{\mathrm{e}}(q)-E_{\mathrm{e}}\left(q^{\prime}\right)\right]}{\partial p^{2}}\right|_{q=q^{\prime}}\left(E_{\mathrm{e}}(q)-E_{\mathrm{e}}\left(q^{\prime}\right)\right)^{2} \\
= & \delta\left(p-p^{\prime}\right)+\frac{\partial \delta\left(p-p^{\prime}\right)}{\partial p^{\prime}} \mathcal{D}_{\left(\frac{q}{m_{\mathrm{e}}}\right)}+\frac{\partial \delta\left(p-p^{\prime}\right)}{\partial p^{\prime}} \mathcal{D}_{\left(\frac{p}{m_{\mathrm{e}}}\right)}+\frac{1}{2} \mathcal{D}_{\left(\frac{q}{m_{\mathrm{e}}}\right)}^{2} \frac{\partial^{2} \delta\left(p-p^{\prime}\right)}{\partial p^{\prime 2}}
\end{aligned}
$$

where

$$
\mathcal{D}_{\left(\frac{q}{m_{\mathrm{e}}}\right)}=\frac{\left(\boldsymbol{p}-\boldsymbol{p}^{\prime}\right) \cdot \boldsymbol{q}}{m_{\mathrm{e}}}, \quad \mathcal{D}_{\left(\frac{p}{m_{\mathrm{e}}}\right)}=\frac{\left(\boldsymbol{p}-\boldsymbol{p}^{\prime}\right)^{2}}{2 m_{\mathrm{e}}} .
$$

Finally, the distribution of the electron can be expanded to

$$
g_{\mathrm{e}}\left(\boldsymbol{q}+\boldsymbol{p}-\boldsymbol{p}^{\prime}\right) \approx g_{\mathrm{e}}(\boldsymbol{q})+\frac{\partial g_{\mathrm{e}}}{\partial \boldsymbol{q}} \cdot\left(\boldsymbol{p}-\boldsymbol{p}^{\prime}\right)+\frac{1}{2}\left(p^{i}-p^{i}\right) \frac{\partial^{2} g_{\mathrm{e}}}{\partial q^{i} \partial q^{j}}\left(p^{j}-p^{\prime j}\right)
$$

We assume that the electrons are kept in thermal equilibrium and in the Boltzmann distribution:

$$
g_{\mathrm{e}}(\boldsymbol{q})=n_{\mathrm{e}}\left(\frac{2 \pi}{m_{\mathrm{e}} T_{\mathrm{e}}}\right)^{3 / 2} \exp \left[-\frac{\left(\boldsymbol{q}-m_{\mathrm{e}} \boldsymbol{v}_{\boldsymbol{e}}\right)^{2}}{2 m_{\mathrm{e}} T_{\mathrm{e}}}\right],
$$

where $v_{e}$ is the bulk velocity of electrons. The derivatives of the distribution function with respect to the momentum are given as

$$
\begin{aligned}
\frac{\partial g_{\mathrm{e}}}{\partial q^{i}} & =-g_{\mathrm{e}} \frac{q_{i}-m_{\mathrm{e}} v_{\mathrm{e} i}}{m_{\mathrm{e}} T_{\mathrm{e}}} \\
\frac{\partial^{2} g_{\mathrm{e}}}{\partial q^{i} \partial q^{j}} & =-\frac{\partial g_{\mathrm{e}}}{\partial q^{j}} \frac{q^{i}-m_{\mathrm{e}} v_{\mathrm{e}}^{i}}{m_{\mathrm{e}} T_{\mathrm{e}}}-g_{\mathrm{e}} \frac{\delta^{i j}}{m_{\mathrm{e}} T_{\mathrm{e}}}
\end{aligned}
$$


By substituting the above equations, Eq. (19) is written as

$$
g_{\mathrm{e}}\left(\boldsymbol{q}+\boldsymbol{p}-\boldsymbol{p}^{\prime}\right) \approx g_{\mathrm{e}}(\boldsymbol{q})\left[1-\mathcal{F}_{\left(\frac{q}{m_{\mathrm{e}}}\right)}+\frac{1}{2} \mathcal{F}_{\left(\frac{q}{m_{\mathrm{e}}}\right)}^{2}-\mathcal{F}_{\left(\frac{p}{m_{\mathrm{e}}}\right)}\right]
$$

where

$$
\mathcal{F}_{\left(\frac{q}{m_{\mathrm{e}}}\right)}=\frac{\boldsymbol{q}-m_{\mathrm{e}} \boldsymbol{v}_{\mathrm{e}}}{m_{\mathrm{e}} T_{\mathrm{e}}} \cdot\left(\boldsymbol{p}-\boldsymbol{p}^{\prime}\right), \quad \mathcal{F}_{\left(\frac{p}{m_{\mathrm{e}}}\right)}=\frac{1}{2} \frac{\left(\boldsymbol{p}-\boldsymbol{p}^{\prime}\right)^{2}}{m_{\mathrm{e}} T_{\mathrm{e}}}
$$

Therefore, we have

$$
\begin{aligned}
& g_{\mathrm{e}}\left(\boldsymbol{q}+\boldsymbol{p}-\boldsymbol{p}^{\prime}\right) f\left(\boldsymbol{p}^{\prime}\right)(1+f(\boldsymbol{p}))-g_{\mathrm{e}}(\boldsymbol{q}) f(\boldsymbol{p})\left(1+f\left(\boldsymbol{p}^{\prime}\right)\right) \\
& =g_{\mathrm{e}}(\boldsymbol{q})\left[f\left(\boldsymbol{p}^{\prime}\right)-f(\boldsymbol{p})\right]-f\left(\boldsymbol{p}^{\prime}\right) g_{\mathrm{e}}(\boldsymbol{q}) \mathcal{F}_{\left(\frac{q}{m_{\mathrm{e}}}\right)}-f\left(\boldsymbol{p}^{\prime}\right) g_{\mathrm{e}}(\boldsymbol{q})\left[\mathcal{F}_{\left(\frac{p}{m_{\mathrm{e}}}\right)}-\frac{1}{2} \mathcal{F}_{\left(\frac{q}{m_{\mathrm{e}}}\right)}^{2}\right] \\
& +g_{\mathrm{e}}(\boldsymbol{q}) f(\boldsymbol{p}) f\left(\boldsymbol{p}^{\prime}\right)\left[-\mathcal{F}_{\left(\frac{q}{m_{\mathrm{e}}}\right)}+\frac{1}{2} \mathcal{F}_{\left(\frac{q}{m_{\mathrm{e}}}\right)}^{2}-\mathcal{F}_{\left(\frac{p}{m_{\mathrm{e}}}\right)}\right] .
\end{aligned}
$$

Combining altogether, we obtain the collision term expanded with respect to the energy transfer as (note that this expansion is not with respect to the density perturbations)

$$
C[f]=\frac{3}{2} \pi^{2} \frac{a \sigma_{T}}{p} \int \frac{d^{3} p^{\prime}}{(2 \pi)^{3} p^{\prime}} \int \frac{d^{3} q}{(2 \pi)^{3}}[(0 \text { th order })+(1 \text { st order })+(2 \text { nd order })]
$$

where

0th order term:

$$
\mathcal{M}_{0} \delta\left(p-p^{\prime}\right) g_{\mathrm{e}}(\boldsymbol{q})\left[f\left(\boldsymbol{p}^{\prime}\right)-f(\boldsymbol{p})\right],
$$

1st order terms:

$$
\begin{aligned}
& \mathcal{M}_{0} g_{\mathrm{e}}(\boldsymbol{q})\left[-\delta\left(p-p^{\prime}\right) f\left(\boldsymbol{p}^{\prime}\right) \mathcal{F}_{\left(\frac{q}{m_{\mathrm{e}}}\right)}+\frac{\partial \delta\left(p-p^{\prime}\right)}{\partial p^{\prime}}\left[f\left(\boldsymbol{p}^{\prime}\right)-f(\boldsymbol{p})\right] \mathcal{D}_{\left(\frac{q}{m_{\mathrm{e}}}\right)}\right] \\
& +\mathcal{M}_{\left(\frac{q}{m_{\mathrm{e}}}\right)} g_{\mathrm{e}}(\boldsymbol{q}) \delta\left(p-p^{\prime}\right)\left[f\left(\boldsymbol{p}^{\prime}\right)-f(\boldsymbol{p})\right]
\end{aligned}
$$

2nd order terms:

$$
\begin{aligned}
& \mathcal{M}_{0} g_{\mathrm{e}}(\boldsymbol{q})\left[-\delta\left(p-p^{\prime}\right) f\left(\boldsymbol{p}^{\prime}\right)\left(\mathcal{F}_{\left(\frac{p}{m_{\mathrm{e}}}\right)}-\frac{1}{2} \mathcal{F}_{\left(\frac{q}{m_{\mathrm{e}}}\right)}^{2}\right)+\frac{1}{2} \frac{\partial^{2} \delta\left(p-p^{\prime}\right)}{\partial p^{\prime 2}} \mathcal{D}_{\left(\frac{q}{m_{\mathrm{e}}}\right)}^{2}\left[f\left(\boldsymbol{p}^{\prime}\right)-f(\boldsymbol{p})\right]\right. \\
& \left.+\frac{\partial \delta\left(p-p^{\prime}\right)}{\partial p^{\prime}} \mathcal{D}_{\left(\frac{p}{m_{\mathrm{e}}}\right)}\left[f\left(\boldsymbol{p}^{\prime}\right)-f(\boldsymbol{p})\right]-\frac{\partial \delta\left(p-p^{\prime}\right)}{\partial p^{\prime}} \mathcal{D}_{\left(\frac{q}{m_{\mathrm{e}}}\right)} f\left(\boldsymbol{p}^{\prime}\right) \mathcal{F}_{\left(\frac{q}{m_{\mathrm{e}}}\right)}\right] \\
& +\mathcal{M}_{\left(\frac{q}{m_{\mathrm{e}}}\right)} g_{\mathrm{e}}(\boldsymbol{q})\left[-\delta\left(p-p^{\prime}\right) f\left(\boldsymbol{p}^{\prime}\right) \mathcal{F}_{\left(\frac{q}{m_{\mathrm{e}}}\right)}+\frac{\partial \delta\left(p-p^{\prime}\right)}{\partial p^{\prime}} \mathcal{D}_{\left(\frac{q}{m_{\mathrm{e}}}\right)}\left[f\left(\boldsymbol{p}^{\prime}\right)-f(\boldsymbol{p})\right]\right] \\
& +\mathcal{M}_{0} g_{\mathrm{e}}(\boldsymbol{q}) f(\boldsymbol{p}) f\left(\boldsymbol{p}^{\prime}\right)\left[-\frac{\partial \delta\left(p-p^{\prime}\right)}{\partial p^{\prime}} \mathcal{F}_{\left(\frac{q}{m_{\mathrm{e}}}\right)} \mathcal{D}_{\left(\frac{q}{m_{\mathrm{e}}}\right)}+\left(\frac{1}{2} \mathcal{F}_{\left(\frac{q}{m_{\mathrm{e}}}\right)}^{2}-\mathcal{F}_{\left(\frac{p}{m_{\mathrm{e}}}\right)}\right) \delta\left(p-p^{\prime}\right)\right] \\
& -g_{\mathrm{e}}(\boldsymbol{q}) f(\boldsymbol{p}) f\left(\boldsymbol{p}^{\prime}\right) \mathcal{M}_{\left(\frac{q}{m_{\mathrm{e}}}\right)} \mathcal{F}_{\left(\frac{q}{m_{\mathrm{e}}}\right)} \delta\left(p-p^{\prime}\right) .
\end{aligned}
$$

From now on, we omit the second-order terms. These terms are not only much smaller than the first-order terms but also may not contribute to the Euler equation at all (see, Ref. [47]). Evaluating the first moment of the above collision term, we obtain the Compton scattering term in the Euler equation (37) as

$$
\begin{aligned}
C_{i}^{\mathrm{e} \gamma} & =-\int \frac{d^{3} p}{(2 \pi)^{3}} p_{i} C[f] \\
& =-\frac{4 a n_{\mathrm{e}} \sigma_{T}}{3} \rho_{\gamma}\left[\left(v_{\mathrm{e} i}-v_{\gamma i}\right)+\frac{3}{4} v_{\mathrm{e} j} \Pi_{\gamma i}{ }^{j}\right]
\end{aligned}
$$

where the product of the velocity of electrons and anisotropic stress of photons in Eq. (30) is the anisotropic part of "radiation drag" in the context of the radiation hydrodynamics. The radiation drag is originated by the electron motion in anisotropic radiation fields with absorptions and emissions. The velocity of electrons $v_{\mathrm{e} i}$ obeys the Euler 
equation given in the next section, and the velocity and anisotropic stress of photons, $v_{\gamma i}$ and $\Pi_{\gamma i}{ }^{j}$, obey the Boltzmann equation. We will show the explicit equations for electrons and photons in Sec. IIIA And we will show the anisotropic stress of photons can be written in terms of the brightness function, which is also defined in Sec. IIIA.

Here moments of the distribution functions are given by

$$
\begin{aligned}
& \int \frac{d^{3} p}{(2 \pi)^{3}} p f_{\gamma}(\boldsymbol{p})=\rho_{\gamma} \\
& \int \frac{d^{3} p}{(2 \pi)^{3}} p_{i} f_{\gamma}(\boldsymbol{p})=\frac{4}{3} \rho_{\gamma} v_{\gamma i} \\
& \int \frac{d^{3} q}{(2 \pi)^{3}} g_{\mathrm{e}}(\boldsymbol{q})=n_{\mathrm{e}} \\
& \int \frac{d^{3} q}{(2 \pi)^{3}} q_{i} g_{\mathrm{e}}(\boldsymbol{q})=\rho_{e} v_{\mathrm{e} i}, \\
& \int \frac{d^{3} p}{(2 \pi)^{3}} p^{-1} p_{i} p_{j} f_{\gamma}(\boldsymbol{p})=\rho_{\gamma} \Pi_{\gamma i j}+\frac{1}{3} \rho_{\gamma} \delta_{i j},
\end{aligned}
$$

where $\rho_{\gamma}$ and $\rho_{\mathrm{e}}\left(=m_{\mathrm{e}} n_{\mathrm{e}}\right)$ are energy densities of photons and electrons, $v_{\gamma}^{i}$ and $v_{\mathrm{e}}^{i}$ are their bulk three velocities defined by $v^{i} \equiv u^{i} / u^{0}$, and $\Pi_{\gamma}^{i j}$ is anisotropic stress of photons. Hereafter we use the relative velocity between photons and electrons as $\delta v_{\gamma \mathrm{b} i} \equiv v_{\gamma i}-v_{\mathrm{e} i}$. It should be noted that the collision term (30) was obtained nonperturbatively with respect to density perturbations [37].

\section{B. Evolution equations of magnetic fields}

Now we obtain the Euler equations for protons and electrons as

$$
\begin{aligned}
& m_{\mathrm{p}} n u_{\mathrm{p}}^{\mu} u_{p i ; \mu}-e n u_{\mathrm{p}}^{\mu} F_{i \mu}=0, \\
& m_{\mathrm{e}} n u_{\mathrm{e}}^{\mu} u_{\mathrm{e} i ; \mu}+e n u_{\mathrm{e}}^{\mu} F_{i \mu}=\frac{4 \sigma_{T} \rho_{\gamma} a n}{3}\left[\delta v_{\gamma \mathrm{b} i}-\frac{3}{4} v_{\mathrm{e} j} \Pi_{\gamma i}{ }^{j}\right],
\end{aligned}
$$

where $m_{\mathrm{p}}$ is the proton mass. Here, we ignore the pressures of proton and electron fluids. In addition, the Coulomb collision term is neglected as explained below Eq. (5). Note that the collision term was not evaluated in a manifestly covariant way. Here the left-hand side in Eqs. (36) and (37) should be evaluated in a conformal coordinate system. We also assumed the local charge neutrality: $n=n_{\mathrm{e}} \sim n_{\mathrm{p}}$. In the case without electromagnetic fields $\left(F_{i \mu}=0\right)$, the sum of the equations (36) and (37) gives the Euler equation for the baryons in the standard perturbation theory. On the other hand, subtracting Eq. (36) multiplied by $m_{\mathrm{e}}$ from Eq. (37) multiplied by $m_{\mathrm{p}}$, we obtain

$$
\begin{aligned}
& -\frac{m_{\mathrm{p}} m_{\mathrm{e}}}{e}\left[n u^{\mu}\left(\frac{j_{i}}{n}\right)_{; \mu}+j^{\mu}\left(\frac{m_{\mathrm{p}}-m_{\mathrm{e}}}{m_{\mathrm{p}}+m_{\mathrm{e}}} \frac{j_{i}}{e n}-u_{i}\right)_{; \mu}\right]+e n\left(m_{\mathrm{p}}+m_{\mathrm{e}}\right) u^{\mu} F_{i \mu}-\left(m_{\mathrm{p}}-m_{\mathrm{e}}\right) j^{\mu} F_{i \mu} \\
& =\frac{4 m_{\mathrm{p}} \rho_{\gamma} a n \sigma_{T}}{3}\left[\delta v_{\gamma \mathrm{b} i}-\frac{3}{4} v_{\mathrm{e} j} \Pi_{\gamma i}{ }^{j}\right]
\end{aligned}
$$

where $u^{\mu}$ and $j^{\mu}$ are the center-of-mass 4 -velocity of the proton and electron fluids and the net electric current, respectively, defined as

$$
\begin{aligned}
u^{\mu} & \equiv \frac{m_{\mathrm{p}} u_{\mathrm{p}}^{\mu}+m_{\mathrm{e}} u_{\mathrm{e}}^{\mu}}{m_{\mathrm{p}}+m_{\mathrm{e}}}, \\
j^{\mu} & \equiv \operatorname{en}\left(u_{\mathrm{p}}^{\mu}-u_{\mathrm{e}}^{\mu}\right) .
\end{aligned}
$$

Employing the Maxwell equations $F_{; \nu}^{\mu \nu}=j^{\mu}$, we see that the quantities in the square bracket in the left-hand side of Eq. (38) is suppressed at the recombination epoch, compared to the second term, by a factor 68]

$$
\frac{c^{2}}{L^{2} \omega_{\mathrm{p}}^{2}} \sim 3 \times 10^{-40}\left(\frac{10^{3} \mathrm{~cm}^{-3}}{n}\right)\left(\frac{1 \mathrm{Mpc}}{L}\right)^{2},
$$

where $c$ is the speed of light, $L$ is a characteristic length of the system and $\omega_{\mathrm{p}}=\sqrt{4 \pi n e^{2} / m_{\mathrm{e}}}$ is the plasma frequency. 
The third term in the left-hand side of Eq. (38), i.e., $\left(m_{\mathrm{p}}-m_{\mathrm{e}}\right) j^{\mu} F_{i \mu}$, is the Hall term which can also be neglected because the Coulomb coupling between protons and electrons is so tight that $\left|u^{i}\right| \gg\left|u_{\mathrm{p}}^{i}-u_{\mathrm{e}}^{i}\right|$. Then we obtain a generalized Ohm's law:

$$
u^{\mu} F_{i \mu}=\frac{4 \sigma_{T} \rho_{\gamma} a}{3 e}\left[\delta v_{\gamma \mathrm{b} i}-\frac{3}{4} v_{\mathrm{e} j} \Pi_{\gamma i}^{j}\right] \equiv C_{i} .
$$

Now we derive the evolution equation for the magnetic field, which can be obtained from the Bianchi identities $F_{[\mu \nu, \lambda]}=0$, as

$$
\begin{aligned}
0 & =\frac{3}{2} \epsilon^{i j k} u^{\mu} F_{[j k, \mu]} \\
& =u^{\mu} \mathcal{B}^{i}{ }_{, \mu}-\epsilon^{i j k}\left(C_{j, k}+\frac{u^{0}{ }_{, j}}{u^{0}} C_{k}\right)-\left(u^{i}{ }_{, j} \mathcal{B}^{j}-u_{, j}^{j} \mathcal{B}^{i}\right)+\frac{u^{0}{ }_{, j}}{u^{0}}\left(\mathcal{B}^{j} u^{i}-\mathcal{B}^{i} u^{j}\right),
\end{aligned}
$$

where $\epsilon^{i j k}$ is the Levi-Cività tensor and $\mathcal{B}^{i} \equiv\left(a^{2} B^{i}\right)=\epsilon^{i j k} F_{j k} / 2$ is the magnetic field in the comoving frame 69. We will now expand the photon energy density, fluid velocities, and photon anisotropic stress with respect to the density perturbation as

$$
\begin{aligned}
& \rho_{\gamma}\left(t, x_{i}\right)=\rho_{\gamma}^{(0)}(t)+\rho_{\gamma}^{(1)}\left(t, x_{i}\right)+\cdots, \quad u^{0}\left(t, x_{i}\right)=a(t)^{-1}+u^{(1) 0}\left(t, x_{i}\right)+\cdots \\
& u^{i}\left(t, x_{i}\right)=u^{(1) i}\left(t, x_{i}\right)+\frac{1}{2} u^{(2) i}\left(t, x_{i}\right)+\cdots, \quad v_{i}\left(t, x_{i}\right)=v_{i}^{(1)}\left(t, x_{i}\right)+\frac{1}{2} v_{i}^{(2)}\left(t, x_{i}\right)+\cdots \\
& \Pi_{\gamma}^{i j}\left(t, x_{i}\right)=\Pi_{\gamma}^{(1) i j}\left(t, x_{i}\right)+\cdots,
\end{aligned}
$$

where the superscripts $(0),(1)$, and (2) denote the order of expansion and $t$ is the cosmic time. Remembering that $\mathcal{B}^{i}$ is a second-order quantity, we see that all terms involving $\mathcal{B}^{i}$ in Eq. (43), other than the first term, can be neglected. Thus we obtain

$$
\begin{aligned}
\frac{d \mathcal{B}^{i}}{d t} & \sim \epsilon^{i j k}\left(C_{j, k}+\frac{u_{, j}^{0}}{u^{0}} C_{k}\right) \\
& =\frac{4 \sigma_{T} \rho_{\gamma}^{(0)} a}{3 e} \epsilon^{i j k}\left[\frac{1}{2} \delta v_{\gamma \mathrm{b} j, k}^{(2)}-\delta_{\gamma, j}^{(1)} \delta v_{\gamma \mathrm{b} k}^{(1)}-\frac{3}{4}\left(v_{\mathrm{e} l}^{(1)} \Pi_{\gamma j}^{(1) l}\right)_{, k}\right]
\end{aligned}
$$

where we used the density contrast of photons, $\delta_{\gamma, k}^{(1)} \equiv \rho_{\gamma, k}^{(1)} / \rho_{\gamma}^{(0)}$. Further, we employed the fact that there is no vorticity in the linear order: $\epsilon^{i j k} v_{j, k}^{(1)}=0$. It should be noted that the velocity of electron fluid can be approximated to the center-of-mass velocity at this order, $v_{\mathrm{e}}^{(1) i} \sim v_{\mathrm{b}}^{(1) i}$. The physical meaning of this equation is that electrons gain (or lose) their momentum through scatterings due to the relative velocity to photons, and the anisotropic stress of photons. The momentum transfer from the photons ensures the velocity difference between electrons and protons, and thus eventually generates magnetic fields. We found that the contribution from the curvature perturbation is always much smaller than that from the density contrast of photons. Furthermore the tensor perturbation, i.e., primordial gravitational waves, is subdominant comparing with the scalar perturbation in the current observations 70 . 72 . Therefore, we have omitted the curvature perturbation and the tensor perturbation in Eq. (45) when considering the evolution of magnetic fields. Equation (45) shows that the magnetic field cannot be generated in the first order. The right-hand side of Eq. (45) contains two types of source terms, i.e., a purely second-order term and those that consist of the products of first order quantities.

The first term in Eq. (45) is exactly the same as that discussed in [73]. They have estimated the amplitude of magnetic fields from these terms by considering typical values at recombination. Here, we solve the equation numerically and obtain a robust prediction of the amplitude of magnetic fields in the standard $\Lambda$ CDM cosmology.

\section{Scalar, vector, and tensor decomposition}

We devote this subsection to rewriting the evolution equation of magnetic fields in the context of the scalar, vector, and tensor decomposition approach. In the standard cosmological perturbation theory, the perturbations can be 
decomposed into three modes, i.e., scalar, vector, and tensor modes [74] as

$$
\begin{aligned}
\omega_{i}(\boldsymbol{k}) & =\omega_{0}(\boldsymbol{k}) O_{i}^{(0)}(\hat{\boldsymbol{k}})+\sum_{\lambda= \pm 1} \omega_{\lambda}(\boldsymbol{k}) O_{i}^{(\lambda)}(\hat{\boldsymbol{k}}), \\
\chi_{i j}(\boldsymbol{k}) & =\chi_{\mathrm{iso}}(\boldsymbol{k}) \delta_{i j}+\chi_{0}(\boldsymbol{k}) O_{i j}^{(0)}(\hat{\boldsymbol{k}})+\sum_{\lambda= \pm 1} \chi_{\lambda}(\boldsymbol{k}) O_{i j}^{(\lambda)}(\hat{\boldsymbol{k}})+\sum_{\sigma= \pm 2} \chi_{\sigma}(\boldsymbol{k}) O_{i j}^{(\sigma)}(\hat{\boldsymbol{k}}),
\end{aligned}
$$

where $O_{i}^{(m)}$ and $O_{i j}^{(m)}$ are the orthogonal bases for Fourier modes with wave number $\boldsymbol{k} \equiv k \hat{\boldsymbol{k}}$ and $m=0, \pm 1$, and \pm 2 represent the scalar, vector, and tensor modes, respectively. Note that, in our notations, the normalization is different from that adopted in Ref. [74]. These inverses are given as

$$
\begin{aligned}
\omega_{0}(\boldsymbol{k}) & =-O_{i}^{(0)}(\hat{\boldsymbol{k}}) \omega_{i}(\boldsymbol{k}), \quad \omega_{\lambda}(\boldsymbol{k})=-O_{i}^{(-\lambda)}(\hat{\boldsymbol{k}}) \omega_{i}(\boldsymbol{k}), \\
\chi_{0}(\boldsymbol{k}) & =\frac{3}{2} O_{i j}^{(0)}(\hat{\boldsymbol{k}}) \chi_{i j}(\boldsymbol{k}), \quad \chi_{\lambda}(\boldsymbol{k})=-2 O_{i j}^{(-\lambda)}(\hat{\boldsymbol{k}}) \chi_{i j}(\boldsymbol{k}), \quad \chi_{\sigma}(\boldsymbol{k})=\frac{2}{3} O_{i j}^{(-\sigma)}(\hat{\boldsymbol{k}}) \chi_{i j}(\boldsymbol{k}) .
\end{aligned}
$$

In this notation, magnetic fields can be decomposed into the scalar and vector modes, i.e., $\mathcal{B}_{i}=\mathcal{B}_{0} O_{i}^{(0)}+$ $\sum_{\lambda= \pm 1} \mathcal{B}_{\lambda} O_{i}^{(\lambda)}$. When we pull out the scalar mode from Eq. (45), we find that the right hand side of Eq. (45) vanishes, namely,

$$
\frac{d \mathcal{B}_{0}}{d t}=0
$$

This is because magnetic fields consist of rotation of the vector potential, or in other words, magnetic fields do not have the scalar component. In contrast, the vector mode for Eq. (45) is given by

$$
\begin{aligned}
\frac{d \mathcal{B}_{\lambda}(\boldsymbol{k})}{d t}= & \frac{4 \sigma_{T} \rho_{\gamma}^{(0)} a}{3 e}(\lambda k)\left[-\frac{1}{2} \delta v_{\gamma \mathrm{b} \lambda}^{(2)}(\boldsymbol{k})+\int \frac{d^{3} k_{1}}{(2 \pi)^{3}} \delta v_{\gamma \mathrm{b} 0}^{(1)}\left(k_{1}\right) \delta_{\gamma}^{(1)}\left(k_{2}\right) \sqrt{\frac{4 \pi}{3}} Y_{1, \lambda}^{*}\left(\hat{\boldsymbol{k}}_{1}\right)\right. \\
& \left.-\int \frac{d^{3} k_{1}}{(2 \pi)^{3}} \frac{5}{4} v_{\mathrm{b} 0}^{(1)}\left(k_{1}\right) \Pi_{\gamma 0}^{(1)}\left(k_{2}\right) \mathcal{Y}_{1, \lambda}^{1,2}\left(\hat{\boldsymbol{k}}_{1}, \hat{\boldsymbol{k}}_{2}\right)\right],
\end{aligned}
$$

where we define the function $\mathcal{Y}_{\ell, m}^{\ell_{1}, \ell_{2}}\left(\hat{\boldsymbol{k}}_{1}, \hat{\boldsymbol{k}}_{2}\right)$ as

$$
\mathcal{Y}_{\ell, m}^{\ell_{1}, \ell_{2}}\left(\hat{\boldsymbol{k}}_{1}, \hat{\boldsymbol{k}}_{2}\right) \equiv(-1)^{m}(2 \ell+1) \sum_{m_{1}, m_{2}}\left(\begin{array}{ccc}
\ell_{1} & \ell_{2} & \ell \\
0 & 0 & 0
\end{array}\right)\left(\begin{array}{ccc}
\ell_{1} & \ell_{2} & \ell \\
m_{1} & m_{2} & -m
\end{array}\right) \sqrt{\frac{4 \pi}{2 \ell_{1}+1}} Y_{\ell_{1}, m_{1}}^{*}\left(\hat{\boldsymbol{k}}_{1}\right) \sqrt{\frac{4 \pi}{2 \ell_{2}+1}} Y_{\ell_{2}, m_{2}}^{*}\left(\hat{\boldsymbol{k}}_{2}\right) .
$$

where $Y_{\ell, m}(\hat{\boldsymbol{k}})$ is the spherical harmonics. In the above equation, the multipoles should satisfy the condition that $\ell_{1}+\ell_{2}+\ell=$ even because of a property of the Wigner-3j symbol. Furthermore, the triangle condition, $\boldsymbol{k}_{2}=\boldsymbol{k}-\boldsymbol{k}_{1}$, is ensured by the delta function. Throughout this paper, we keep $\boldsymbol{k}_{2}$ in equations for simplicity of presentation even after being integrating out. This function obeys the following relations,

$$
\mathcal{Y}_{\ell, m}^{\ell_{1}, \ell_{2}}\left(\hat{\boldsymbol{k}}_{1}, \hat{\boldsymbol{k}}_{2}\right)=\mathcal{Y}_{\ell, m}^{\ell_{2}, \ell_{1}}\left(\hat{\boldsymbol{k}}_{2}, \hat{\boldsymbol{k}}_{1}\right), \quad \mathcal{Y}_{\ell, m}^{\ell_{1}, 0}\left(\hat{\boldsymbol{k}}_{1}, \hat{\boldsymbol{k}}_{2}\right)=\sqrt{\frac{4 \pi}{2 \ell+1}} Y_{\ell, m}^{*}\left(\hat{\boldsymbol{k}}_{1}\right) \delta_{\ell, \ell_{1}}, \quad \mathcal{Y}_{\ell, m}^{0, \ell_{2}}\left(\hat{\boldsymbol{k}}_{1}, \hat{\boldsymbol{k}}_{2}\right)=\sqrt{\frac{4 \pi}{2 \ell+1}} Y_{\ell, m}^{*}\left(\hat{\boldsymbol{k}}_{2}\right) \delta_{\ell, \ell_{2}} .
$$

To obtain the magnetic fields from Eq. (51), we need to know the second-order relative velocity between baryons and photons for the vector mode. In the next section, we show the formulation of the second-order Einstein-Boltzmann system.

\section{SECOND-ORDER EINSTEIN-BOLTZMANN SYSTEM}

In this section, we derive the perturbed equations of the Einstein-Boltzmann system up to the second order. Throughout this paper, we work in the Poisson gauge of which the line element is

$$
d s^{2}=a^{2}(\eta)\left[-e^{2 \Psi} d \eta^{2}+2 \omega_{i} d \eta d x^{i}+\left(e^{-2 \Phi} \delta_{i j}+\chi_{i j}\right) d x^{i} d x^{j}\right] .
$$

Under the Poisson gauge, the gauge conditions $\omega^{i}{ }_{i}=\chi^{i j}{ }_{j}=0$ and the traceless condition $\chi^{i}{ }_{i}=0$ are imposed on $\omega_{i}$ and $\chi_{i j}$. We expand the metric perturbations as $\Psi=\Psi^{(1)}+\frac{1}{2} \Psi^{(2)}, \Phi=\Phi^{(1)}+\frac{1}{2} \Phi^{(2)}, \omega_{i}=\frac{1}{2} \omega_{i}^{(2)}$, and $\chi_{i j}=\frac{1}{2} \chi_{i j}^{(2)}$, where we neglect the first-order vector and tensor perturbations. The first-order vector perturbation is neglected in the standard cosmology because the vector perturbation has only a decaying mode in the first-order perturbation theory. The first-order tensor perturbation is observationally shown to be subdominant compared with the scalar one at first order [71]. 


\section{A. Boltzmann equation}

From Eq. (51), full computation of magnetic fields needs to evaluate the relative velocity between baryons and photons in the second-order perturbation theory. The velocity perturbations of the two fluids are described by the Boltzmann equation.

The Boltzmann equation with binary collisions can be written as

$$
\frac{d f}{d \lambda}\left(x^{\mu}, P^{\mu}\right)=\tilde{C}[f],
$$

where $\lambda$ is the affine parameter and $\tilde{C}[f]$ is the collision term, i.e., in the case of the photon distribution function, the Thomson interaction between photons and electrons. Note that protons and electrons interact with each other through the Coulomb interaction. We can treat proton and electron fluids as a single component since these particles couple strongly by the Coulomb interaction. For the Boltzmann equation for dark matter or neutrinos, the collision term must vanish.

To calculate the perturbed Boltzmann equation, it is useful to change the coordinate system from the Poisson gauge $\left(x^{\mu}, P^{\mu}\right)$ to the local inertial frame $\left(x^{\mu}, p^{\mu}\right)$ [45]. Since we consider the cosmological perturbations up to the second order, the distribution function is expanded as

$$
f(\eta, \boldsymbol{x}, p, \hat{\boldsymbol{n}})=f^{(0)}(\eta, p)+f^{(1)}(\eta, \boldsymbol{x}, p, \hat{\boldsymbol{n}})+\frac{1}{2} f^{(2)}(\eta, \boldsymbol{x}, p, \hat{\boldsymbol{n}}),
$$

where $p$ and $\hat{\boldsymbol{n}}$ are the amplitude and the direction of the photon's momentum, respectively. The zeroth-order distribution function, $f^{(0)}(\eta, p)$, is fixed to the Planck distribution. It is useful to define the brightness function which is given by

$$
\Delta^{(1,2)}(\eta, \boldsymbol{x}, \hat{\boldsymbol{n}})=\frac{\int d p p^{3} f^{(1,2)}(\eta, \boldsymbol{x}, p, \hat{\boldsymbol{n}})}{\int d p p^{3} f^{(0)}(\eta, p)},
$$

where the denominator of the right-hand side is proportional to the mean energy density of photons.

The angle dependence of the brightness function is expanded by the spherical harmonics as

$$
\Delta^{(1,2)}(\eta, \boldsymbol{x}, \hat{\boldsymbol{n}})=\sum_{\ell} \sum_{m=-\ell}^{\ell} \Delta_{\ell, m}^{(1,2)}(\eta, \boldsymbol{x})(-i)^{\ell} \sqrt{\frac{4 \pi}{2 \ell+1}} Y_{\ell, m}(\hat{\boldsymbol{n}}) .
$$

The coefficients $\Delta_{\ell, m}^{(1)}$ are related to the density perturbation, velocity, and anisotropic stress for photons as $\Delta_{0,0}^{(1)}=\delta_{\gamma}^{(1)}$, $\Delta_{1,0}^{(1)}=4 v_{\gamma 0}^{(1)}$, and $\Delta_{2,0}^{(1)}=5 \Pi_{\gamma 0}^{(1)}$, respectively, as is shown Eqs. (31)-(35). The Boltzmann equation of photons in terms of $\Delta_{\ell, m}^{(1,2)}$ at first- and second-order is written as

$$
\dot{\Delta}_{\ell, m}^{(1,2)}+k\left[\frac{c_{\ell+1, m}}{2 \ell+3} \Delta_{\ell+1, m}^{(1,2)}-\frac{c_{\ell, m}}{2 \ell-1} \Delta_{\ell-1, m}^{(1,2)}\right]=S_{\ell, m}^{(1,2)},
$$

where $c_{\ell, m} \equiv \sqrt{\ell^{2}-m^{2}}$. A dot represents a derivative with respect to the conformal time $\eta$ and here we have translated from real space to Fourier space. The source term $S_{\ell, m}^{(2)}$ can be expressed as

$$
S_{\ell, m}^{(2)}(\boldsymbol{k}, \eta)=\mathcal{C}_{\ell, m}^{(2)}(\boldsymbol{k}, \eta)+\mathcal{G}_{\ell, m}^{(2)}(\boldsymbol{k}, \eta)
$$

Here, $\mathcal{C}_{\ell, m}^{(2)}$ is the collision term that is proportional to $\dot{\tau}_{c}$, where $\dot{\tau}_{c}$ is the differential optical depth which is defined by the number density of the electron $n_{e}$, scale factor $a$, and the Thomson scattering cross-section $\sigma_{\mathrm{T}}$ as $\dot{\tau}_{c}=-a n_{e} \sigma_{\mathrm{T}}$, and $\mathcal{G}_{\ell, m}^{(2)}$ denotes the gravitational effects, i.e., the lensing and the redshift terms. The collision term $\mathcal{C}_{\ell, m}^{(2)}$ is related to Eq. (26) as

$$
\mathcal{C}_{\ell, m}^{(2)}(\boldsymbol{k}, \eta)=\int d \Omega_{n}(-i)^{-\ell} \sqrt{\frac{2 \ell+1}{4 \pi}} Y_{\ell, m}^{*}(\hat{\boldsymbol{n}}) \frac{\int d p p^{3} \mathcal{C}^{(2)}(\eta, \boldsymbol{k}, p, \hat{\boldsymbol{n}})}{\int d p p^{3} f^{(0)}(\eta, p)}
$$

where $\mathcal{C}^{(2)}(\eta, \boldsymbol{k}, p, \hat{\boldsymbol{n}})$ is the Fourier transformation of Eq. (26) , while the gravitational effect $\mathcal{G}_{\ell, m}^{(2)}$ is coming from the left-hand side of the Boltzmann equation (55) with the same procedure as obtaining $\mathcal{C}^{(2)}(\eta, \boldsymbol{k}, p, \hat{\boldsymbol{n}})$. In this paper, we 
call $\mathcal{C}_{\ell, m}$ and $\mathcal{G}_{\ell, m}$ the scattering term and the gravitational term, respectively. The explicit forms of $\mathcal{C}_{\ell, m}^{(2)}$ and $\mathcal{G}_{\ell, m}^{(2)}$ are given in our previous study [75].

The source terms of the first-order Boltzmann equation vanish when $m \neq 0$, because we consider only the scalar mode in the first-order perturbations. However, for the second-order perturbations, not only the scalar mode $(m=0)$, but also the vector $(m=\lambda)$ and tensor $(m=\sigma)$ modes arise due to nonlinear couplings, where $\lambda= \pm 1$ and $\sigma= \pm 2$, respectively.

In the case of massless neutrinos, one can set $\dot{\tau}_{c}=0$ in the above equations because massless neutrinos interact with the other fluids only through gravity. We do not write down the hierarchical equation of neutrinos here since it is trivial. The distribution function of neutrinos is also expanded by the spherical harmonics.

\section{B. Tight coupling solutions of the vector mode}

To solve the second-order equations derived in Sec. III A numerically, we should set up the initial condition of each perturbation variable. Thus we first solve the equations analytically with $k \eta \ll 1$ and using the tight coupling approximation, and find the initial condition at sufficiently early time for our numerical calculation.

Deep in the radiation dominated era, photon and baryon fluids are tightly coupled because the opacity $\dot{\tau}_{c}$ is large 57, 58, 76, 77]. Although the photon and baryon fluids would behave as a single fluid, there is a small difference in motion between photon and baryon fluids. For this reason, we can expand the perturbation variables using the tight-coupling parameter which is given by

$$
\epsilon \equiv\left|\frac{k}{\dot{\tau}_{c}}\right| \sim 10^{-2}\left(\frac{k}{1 \mathrm{Mpc}^{-1}}\right)\left(\frac{1+z}{10^{4}}\right)^{-2}\left(\frac{\Omega_{\mathrm{b}} h^{2}}{0.02}\right)^{-1},
$$

where $\Omega_{\mathrm{b}}$ is the baryon density normalized by the critical density, and $h$ is the normalized Hubble constant. In what follows, we derive the tight-coupling solution up to the first order to set the initial condition of photon and baryon fluids at second-order in cosmological perturbations and to calculate the evolution of perturbations in a numerically stable manner.

We expand the cosmological perturbation variables using the tight-coupling parameter $\epsilon$ up to the first order as

$$
\Delta^{(\mathrm{CPT}=1,2)}=\Delta^{(\mathrm{CPT}=1,2, \mathrm{TCA}=\varnothing)}+\Delta^{(\mathrm{CPT}=1,2, \mathrm{TCA}=I)}+\cdots
$$

where the Arabic number and the Roman number represent orders in the cosmological perturbation and the tight coupling expansion, respectively. In the rest of this paper, we focus on the vector mode $m= \pm 1$.

First, the solutions at zeroth order in the tight-coupling expansion, namely, in the tight-coupling limit, are given as

$$
\begin{aligned}
\delta v_{\gamma \mathrm{b} \lambda}^{(2, \varnothing)}(\boldsymbol{k}) & =0 \\
\Delta_{2, \lambda}^{(2, \varnothing)}(\boldsymbol{k}) & =20 \int \frac{d^{3} k_{1}}{(2 \pi)^{3}}\left[v_{\gamma 0}^{(1, \varnothing)}\left(k_{1}\right) v_{\gamma}^{(1, \varnothing)}\left(k_{2}\right)\right] \mathcal{Y}_{2, \lambda}^{1,1}\left(\hat{\boldsymbol{k}}_{1}, \hat{\boldsymbol{k}}_{2}\right) \\
\Delta_{\ell \geq 3, \lambda}^{(2, \varnothing)}(\boldsymbol{k}) & =0
\end{aligned}
$$

where $\boldsymbol{k}_{2}=\boldsymbol{k}-\boldsymbol{k}_{1}$ in Eq. (65). In the tight-coupling limit, the relative velocity between photons and baryons vanishes as well as in the first-order cosmological perturbation theory. However, the anisotropic stress of photons is present due to the quadratic of the photon velocity.

Second, the solutions at first-order in the tight-coupling expansion are given as 


$$
\begin{aligned}
& \frac{1+R}{R} \delta v_{\gamma \mathrm{b} \lambda}^{(2, I)}(\boldsymbol{k})=\frac{\sqrt{3}}{20}\left(\frac{k}{\dot{\tau}_{c}}\right) \Delta_{2, \lambda}^{(2)}(\boldsymbol{k})-\left(\frac{\mathcal{H}}{\dot{\tau}_{c}}\right)\left(\omega_{\lambda}^{(2)}(\boldsymbol{k})+v_{\mathrm{b} \lambda}^{(2)}(\boldsymbol{k})\right) \\
& +\int \frac{d^{3} k_{1}}{(2 \pi)^{3}}\left[-2 \delta v_{\gamma \mathbf{b} 0}^{(1)}\left(k_{1}\right)\left(\delta_{\mathrm{b}}^{(1)}+\delta_{\gamma}^{(1)}+\Psi^{(1)}\right)\left(k_{2}\right)\right] \sqrt{\frac{4 \pi}{3}} Y_{1, \lambda}^{*}\left(\hat{\boldsymbol{k}}_{1}\right) \\
& +\int \frac{d^{3} k_{1}}{(2 \pi)^{3}}\left[\frac{1}{2} v_{\mathrm{b} 0}^{(1)}\left(k_{1}\right) \Pi_{\gamma 0}^{(1)}\left(k_{2}\right)\right] \sqrt{\frac{4 \pi}{3}} Y_{1, \lambda}^{*}\left(\hat{\boldsymbol{k}}_{1}\right) \\
& +\int \frac{d^{3} k_{1}}{(2 \pi)^{3}}\left[2 v_{\gamma 0}^{(1)}\left(k_{1}\right) \delta v_{\gamma \mathrm{b} 0}^{(1)}\left(k_{2}\right)\right] \sqrt{\frac{4 \pi}{3}} Y_{1, \lambda}^{*}\left(\hat{\boldsymbol{k}}_{1}\right) \\
& +\frac{1}{R} \int \frac{d^{3} k_{1}}{(2 \pi)^{3}}\left[-2 \delta v_{\gamma \mathrm{b} 0}^{(1)}\left(k_{1}\right)\left(\delta_{\gamma}^{(1)}+\Psi^{(1)}\right)\left(k_{2}\right)\right] \sqrt{\frac{4 \pi}{3}} Y_{1, \lambda}^{*}\left(\hat{\boldsymbol{k}}_{1}\right) \\
& +\int \frac{d^{3} k_{1}}{(2 \pi)^{3}}\left(\frac{k_{1}}{\dot{\tau}_{c}}\right)\left[-\frac{1}{2} \delta_{\gamma}^{(1)}\left(k_{1}\right)\left(\Psi^{(1)}+\Phi^{(1)}\right)\left(k_{2}\right)-2 \Psi^{(1)}\left(k_{1}\right) \delta_{\gamma}^{(1)}\left(k_{2}\right)\right] \sqrt{\frac{4 \pi}{3}} Y_{1, \lambda}^{*}\left(\hat{\boldsymbol{k}}_{1}\right) \\
& +\int \frac{d^{3} k_{1}}{(2 \pi)^{3}}\left(\frac{k_{2}}{\dot{\tau}_{c}}\right)\left[\frac{1}{2} v_{\gamma 0}^{(1)}\left(k_{1}\right)\left(\delta_{\gamma}^{(1)}+4 \Psi^{(1)}\right)\left(k_{2}\right)\right] \sqrt{\frac{4 \pi}{3}} Y_{1, \lambda}^{*}\left(\hat{\boldsymbol{k}}_{1}\right) \\
& +\int \frac{d^{3} k_{1}}{(2 \pi)^{3}}\left(\frac{k_{1}}{\dot{\tau}_{c}}\right)\left[-\frac{8}{3} v_{\gamma 0}^{(1)}\left(k_{1}\right) \delta_{\gamma}^{(1)}\left(k_{2}\right)\right] \sqrt{\frac{4 \pi}{3}} Y_{1, \lambda}^{*}\left(\hat{\boldsymbol{k}}_{1}\right) \\
& +\int \frac{d^{3} k_{1}}{(2 \pi)^{3}}\left(\frac{k_{2}}{\dot{\tau}_{c}}\right)\left[-\frac{2}{3} v_{\mathrm{b} 0}^{(1)}\left(k_{1}\right) v_{\mathrm{b} 0}^{(1)}\left(k_{2}\right)\right] \sqrt{\frac{4 \pi}{3}} Y_{1, \lambda}^{*}\left(\hat{\boldsymbol{k}}_{1}\right) \\
& +\int \frac{d^{3} k_{1}}{(2 \pi)^{3}}\left[-\frac{15}{4} \Pi_{\gamma 0}^{(1)}\left(k_{1}\right) v_{\mathrm{b} 0}^{(1)}\left(k_{2}\right)\right] \mathcal{Y}_{1, \lambda}^{2,1}\left(\hat{\boldsymbol{k}}_{1}, \hat{\boldsymbol{k}}_{2}\right) \\
& +\frac{1}{R} \int \frac{d^{3} k_{1}}{(2 \pi)^{3}}\left[-\frac{5}{2} \Pi_{\gamma 0}^{(1)}\left(k_{1}\right) v_{\mathrm{b} 0}^{(1)}\left(k_{2}\right)\right] \mathcal{Y}_{1, \lambda}^{2,1}\left(\hat{\boldsymbol{k}}_{1}, \hat{\boldsymbol{k}}_{2}\right) \\
& +\int \frac{d^{3} k_{1}}{(2 \pi)^{3}}\left(\frac{k_{1}}{\dot{\tau}_{c}}\right)\left[-\frac{10}{3} v_{\mathrm{b} 0}^{(1)}\left(k_{1}\right) v_{\mathrm{b} 0}^{(1)}\left(k_{2}\right)\right] \mathcal{Y}_{1, \lambda}^{2,1}\left(\hat{\boldsymbol{k}}_{1}, \hat{\boldsymbol{k}}_{2}\right), \\
& \frac{9}{10} \Delta_{2, \lambda}^{(2, I)}(\boldsymbol{k})=-\left(\frac{k}{\dot{\tau}_{c}}\right) \frac{\sqrt{3}}{3} \Delta_{1, \lambda}^{(2)}(\boldsymbol{k}) \\
& +\int \frac{d^{3} k_{1}}{(2 \pi)^{3}}\left[-9 \Pi_{\gamma 0}^{(1)}\left(k_{1}\right)\left(\delta_{\mathrm{b}}^{(1)}+\Psi^{(1)}\right)\left(k_{2}\right)\right] \sqrt{\frac{4 \pi}{5}} Y_{2, \lambda}^{*}\left(\hat{\boldsymbol{k}}_{1}\right) \\
& +\int \frac{d^{3} k_{1}}{(2 \pi)^{3}}\left(\frac{k_{1}}{\dot{\tau}_{c}}\right)\left[-\frac{16}{3} v_{\gamma 0}^{(1)}\left(k_{1}\right)\left(\Psi^{(1)}+\Phi^{(1)}\right)\left(k_{2}\right)\right] \sqrt{\frac{4 \pi}{5}} Y_{2, \lambda}^{*}\left(\hat{\boldsymbol{k}}_{1}\right) \\
& +\int \frac{d^{3} k_{1}}{(2 \pi)^{3}}\left[18 v_{\gamma 0}^{(1)}\left(k_{1}\right) v_{\gamma 0}^{(1)}\left(k_{2}\right)+8 v_{\gamma 0}^{(1)}\left(k_{1}\right) \delta v_{\gamma \mathrm{b} 0}^{(1)}\left(k_{2}\right)\right] \mathcal{Y}_{2, \lambda}^{1,1}\left(\hat{\boldsymbol{k}}_{1}, \hat{\boldsymbol{k}}_{2}\right) \\
& +\int \frac{d^{3} k_{1}}{(2 \pi)^{3}}\left(\frac{k_{1}}{\dot{\tau}_{c}}\right)\left[\left(10 \delta_{\gamma}^{(1)}\left(k_{1}\right)-8 \Phi^{(1)}\right)\left(k_{1}\right) v_{\gamma}^{(1)}\left(k_{2}\right)\right] \mathcal{Y}_{2, \lambda}^{1,1}\left(\hat{\boldsymbol{k}}_{1}, \hat{\boldsymbol{k}}_{2}\right), \\
& \Delta_{3, \lambda}^{(2, I)}(\boldsymbol{k})=-\left(\frac{k}{\dot{\tau}_{c}}\right) \frac{2 \sqrt{2}}{5} \Delta_{2, \lambda}^{(2)}+15 \int \frac{d^{3} k_{1}}{(2 \pi)^{3}}\left[\Pi_{\gamma}^{(1)}\left(k_{1}\right) v_{\gamma}^{(1)}\left(k_{2}\right)\right] \mathcal{Y}_{3, \lambda}^{2,1}\left(\hat{\boldsymbol{k}}_{1}, \hat{\boldsymbol{k}}_{2}\right), \\
& \Delta_{\ell \geq 4, \lambda}^{(2, I)}(\boldsymbol{k})=0
\end{aligned}
$$

where $R \equiv 3 \rho_{\mathrm{b}}^{(0)} /\left(4 \rho_{\gamma}^{(0)}\right)$. To derive the above solutions, the time derivative of the first-order curvature perturbation $\dot{\Phi}$ is ignored due to the conservation of the curvature perturbation on large scales. In the right-hand side of Eqs. (67), (68), and (69), for simplicity, we omit the superscript for the order of the tight-coupling parameter. Note that the octopole does not vanish and higher multipoles than $\ell=3$ are equal to zero at this order [75]. 


\section{Einstein equation of the vector mode}

In this subsection, we derive the second-order Einstein equation and hereafter we focus on the vector mode only. The evolution equation for the metric perturbation of the vector mode, i.e., $\omega_{\lambda}$ can be derived from the $(i, j)$ component of the Einstein equation as

$$
\begin{aligned}
& a^{2} G^{i}{ }_{j}=e^{2 \Phi}\left(\Phi^{, i}{ }_{, j}-\Psi^{, i}{ }_{, j}\right)+\Phi^{, i} \Phi_{, j}-\Psi^{, i} \Psi_{, j}-\left(\Phi^{, i} \Psi_{, j}+\Phi_{, j} \Psi^{, i}\right) \\
& +\mathcal{H}\left[\dot{\chi}^{i}{ }_{j}-\left(\omega^{i}, j+\omega_{j}{ }^{i}\right)\right]+\frac{1}{2}\left[\ddot{\chi}^{i}{ }_{j}-\left(\dot{\omega}^{i}, j+\dot{\omega}_{j}{ }^{i}\right)-\chi^{i}{ }_{j}{ }^{,}{ }^{a}, a\right] \\
& +(\text { diagonal part }) \delta^{i}{ }_{j}
\end{aligned}
$$

and

$$
\begin{aligned}
T_{\mathrm{r} j}^{i} & =\rho_{\mathrm{r}} \Pi_{\mathrm{r} j}^{i}+(\text { diagonal part }) \delta^{i}{ }_{j}, \\
T_{\mathrm{m} j}^{i} & =\rho_{\mathrm{m}} v_{\mathrm{m} i}^{(1)} v_{\mathrm{m} j}^{(1)}+(\text { diagonal part }) \delta^{i}{ }_{j},
\end{aligned}
$$

where $T_{\mathrm{r} j}^{i}$ and $T_{\mathrm{m} j}^{i}$ represent the energy-momentum tensors of massless (relativistic) particles such as photons and neutrinos, and massive (nonrelativistic) particles such as baryons and dark matter, respectively. The equation of the vector mode is derived by acting the projection operator, $-O_{i j}^{(-\lambda)}(\hat{\boldsymbol{k}})$ which can pull the vector mode, as

$$
\begin{aligned}
\dot{\omega}_{\lambda}^{(2)}(\boldsymbol{k})+2 \mathcal{H} \omega_{\lambda}^{(2)}(\boldsymbol{k})= & \frac{2}{5 \sqrt{3}} \frac{1}{k}\left(8 \pi G a^{2} \rho_{\gamma}^{(0)} \Delta_{2, \lambda}^{(2)}(\boldsymbol{k})+8 \pi G a^{2} \rho_{\nu}^{(0)} \mathcal{N}_{2, \lambda}^{(2)}(\boldsymbol{k})\right) \\
& +\int \frac{d^{3} k_{1}}{(2 \pi)^{3}} 4 k_{1}\left[\Phi^{(1)}\left(k_{1}\right) \Psi^{(1)}\left(k_{2}\right)\right] \sqrt{\frac{4 \pi}{3}} Y_{1, \lambda}^{*}\left(\hat{\boldsymbol{k}}_{1}\right) \\
& -\int \frac{d^{3} k_{1}}{(2 \pi)^{3}} \frac{4}{\sqrt{3}} \frac{k_{1}^{2}}{k}\left[\Phi^{(1)}\left(k_{1}\right) \Phi^{(1)}\left(k_{2}\right)+\Psi^{(1)}\left(k_{1}\right) \Psi^{(1)}\left(k_{2}\right)\right] \sqrt{\frac{4 \pi}{5}} Y_{2, \lambda}^{*}\left(\hat{\boldsymbol{k}}_{1}\right) \\
& +\sum_{\mathrm{s}=\mathrm{b}, \mathrm{dm}} 8 \pi G a^{2} \rho_{\mathrm{s}}^{(0)} \int \frac{d^{3} k_{1}}{(2 \pi)^{3}}\left[\frac{4}{k} v_{\mathrm{s} 0}^{(1)}\left(k_{1}\right) v_{\mathrm{s} 0}^{(1)}\left(k_{2}\right)\right] \sqrt{\frac{4 \pi}{3}} Y_{1,0}^{*}\left(\hat{\boldsymbol{k}}_{1}\right) \sqrt{\frac{4 \pi}{3}} Y_{1, \lambda}^{*}\left(\hat{\boldsymbol{k}}_{2}\right)
\end{aligned}
$$

Note that in the Poisson gauge, the vector metric perturbation is only included in $\omega_{i}$. When we consider the evolution equation up to the first order in the standard cosmology with perfect fluids, the right-hand side of Eq. (74) becomes zero. As a result, the vector mode has only a decaying solution, which is neglected in the linear theory.

\section{COSMOLOGICAL MAGNETIC FIELDS}

In this section, we show the evolutions and spectra of magnetic fields driven by the Harrison mechanism. In the previous studies [60, 61, 63, 64, 78], generated magnetic fields are partially estimated by numerical or analytical ways, and there is a small discrepancy between in Refs. [63] and [64]. In this paper, we build on Ref. [63] and expand the work by including all contributions numerically. The source terms of the magnetic fields consist of three contributions, i.e., $\delta_{\gamma}^{(1)} \delta v_{\gamma \mathrm{b}}^{(1)}, v_{\mathrm{b}}^{(1)} \Pi_{\gamma}^{(1)}$, and $\delta v_{\gamma \mathrm{b}}^{(2)}$, which hereafter we call "the slip term", "the anisotropic stress term" and "the second-order slip term", respectively.

In this paper, we focus on three issues on the generation of magnetic fields at recombination. First, we consider how large is the contribution of the second-order slip term on magnetic fields compared with the contributions of the slip and the anisotropic stress terms. Second, to evaluate the total spectrum of magnetic fields we need to include the cross-correlation terms between the sources, namely, $P_{B} \sim\left\langle\left(B_{2 \text { nd Slip }}+B_{\text {Slip }}+B_{\text {Anis }}\right)^{2}\right\rangle$. The cross terms can be negative and it has the possibility to cancel the generated magnetic fields from each of the source terms. Third, we try to find the cause of the small discrepancy between Refs. 63] and 64]. In the following subsections, we show the evolutions and spectra of magnetic fields and the answers of the above three considerable questions.

\section{A. Configuration in Fourier space}

Before moving to the results, we mention the configuration of Fourier space in carrying out the convolution since all second-order quantities are written by the products of the first-order scalar perturbations. To calculate the secondorder power spectrum, we decompose the second-order variable into the transfer function and the primordial amplitude 
79] as

$$
\Delta^{(2)}(\eta, \boldsymbol{k})=\int \frac{d^{3} k_{1}}{(2 \pi)^{3}} \int \frac{d^{3} k_{2}}{(2 \pi)^{3}} \delta\left(\boldsymbol{k}-\boldsymbol{k}_{1}-\boldsymbol{k}_{2}\right) \Delta_{\mathrm{T}}^{(2)}\left(\eta, \boldsymbol{k}, \boldsymbol{k}_{1}, \boldsymbol{k}_{2}\right) \Phi\left(\boldsymbol{k}_{1}\right) \Phi\left(\boldsymbol{k}_{2}\right)
$$

where $\Delta_{\mathrm{T}}^{(2)}$ and $\Phi$ are the second-order transfer function and the primordial amplitude, respectively. The ensemble average of the variance of the primordial amplitude can be expressed as $\left\langle\Phi^{*}\left(\boldsymbol{k}_{1}\right) \Phi\left(\boldsymbol{k}_{2}\right)\right\rangle=(2 \pi)^{3} P_{\Phi}\left(k_{1}\right) \delta\left(\boldsymbol{k}_{1}-\boldsymbol{k}_{2}\right)$, where $P_{\Phi}(k)$ is the primordial power spectrum determined from cosmological observations such as CMB and large scales structure. In this paper, we use the power-law spectrum as

$$
\frac{k^{3}}{2 \pi^{2}} P_{\Phi}(k)=\frac{4}{9} \mathcal{A}_{s}\left(\frac{k}{k_{0}}\right)^{n_{s}-1},
$$

where the parameters, $\mathcal{A}_{s}$ and $n_{s}$, are the amplitude and the spectral index of primordial perturbations, respectively. We set $\mathcal{A}_{s}=2.4 \times 10^{-9}$ from the WMAP nine-year results [80], and for simplicity, we consider a scale-invariant spectrum, namely, $n_{s}=1.0$.

In this decomposition, the second-order power spectrum can be written as

$$
P_{\Delta^{(2)}}(\eta, \boldsymbol{k})=2 \int \frac{d^{3} k_{1}}{(2 \pi)^{3}}\left[\Delta_{\mathrm{T}}^{(2)}\left(\eta, \boldsymbol{k}, \boldsymbol{k}_{1}, \boldsymbol{k}_{2}\right)\right]^{2} P_{\Phi}\left(k_{1}\right) P_{\Phi}\left(k_{2}\right)
$$

where we can use the symmetry under the exchange of $k_{1}$ and $k_{2}$ without loss of generality to derive the above relation. Note that $\boldsymbol{k}_{2}=\boldsymbol{k}-\boldsymbol{k}_{1}$ should be satisfied implicitly, namely, $\Delta_{\mathrm{T}}^{(2)}\left(\eta, \boldsymbol{k}, \boldsymbol{k}_{1}, \boldsymbol{k}_{2}\right)=\Delta_{\mathrm{T}}^{(2)}\left(\eta, \boldsymbol{k}, \boldsymbol{k}_{1}, \boldsymbol{k}-\boldsymbol{k}_{1}\right)$ and $P_{\Phi}\left(k_{2}\right)=P_{\Phi}\left(\left|\boldsymbol{k}-\boldsymbol{k}_{1}\right|\right)$, as is mentioned before.

We need to solve the Einstein-Boltzmann system in $\left(k, k_{1}, k_{2}\right)$ space. Note that the transfer function is transformed under the rotation of $\phi$ as

$$
\Delta_{\mathrm{T}}^{(2)}\left(\eta, \boldsymbol{k}, \boldsymbol{k}_{1}, \boldsymbol{k}_{2}\right) \longrightarrow \Delta_{\mathrm{T}}^{(2)}\left(\eta, \boldsymbol{k}, \boldsymbol{k}_{1}, \boldsymbol{k}_{2}\right) e^{i m \phi}
$$

In practice, we take $\phi=\theta=0, \phi_{1}=0$, and $\phi_{2}=\pi$ for $\boldsymbol{k}, \boldsymbol{k}_{1}$, and $\boldsymbol{k}_{2}$, respectively. In other words, the transfer function under the exchange of $k_{1}$ and $k_{2}$ transforms as

$$
\Delta_{\mathrm{T}}^{(2)}\left(\eta, \boldsymbol{k}, \boldsymbol{k}_{1}, \boldsymbol{k}_{2}\right)=(-1)^{m} \Delta_{\mathrm{T}}^{(2)}\left(\eta, \boldsymbol{k}, \boldsymbol{k}_{2}, \boldsymbol{k}_{1}\right)
$$

It is very interesting that for the case of the scalar and tensor modes, the dominant contribution comes from near the $k \sim k_{1} \sim k_{2}$. Because the transfer functions of the scalar $(m=0)$ and tensor $(m= \pm 2)$ modes are symmetric under the exchange of $k_{1}$ and $k_{2}$. On the other hand, the vector mode does not have dominant contribution near the $k \sim k_{1} \sim k_{2}$ since the transfer function of the vector mode is antisymmetric under the exchange of $k_{1}$ and $k_{2}$.

In the vector mode, we can naively consider three configurations of the triangle $\boldsymbol{k}_{1} \boldsymbol{k}_{1}+\boldsymbol{k}_{2}$ that contribute to the power spectrum on superhorizon scales, namely, the one where both $k_{1}$ and $k_{2}$ are on superhorizon scales, where $k_{1}$ $\left(k_{2}\right)$ is at superhorizon scales while $k_{2}\left(k_{1}\right)$ is at subhorizon scales, and where both $k_{1}$ and $k_{2}$ are at subhorizon scales. When both $k_{1}$ and $k_{2}$ are at subhorizon scales, the square of the primordial power spectrum in Eq. (77) does not contribute on the power spectrum because of the antisymmetric nature of the vector transfer function. In the vector mode, therefore, we need a careful treatment of how to sample wave numbers in $\left(k, k_{1}, k_{2}\right)$ space. This difficulty does not arise in the second-order scalar and tensor modes calculations because the transfer functions of the scalar and tensor modes have a symmetry under the exchange of $k_{1}$ and $k_{2}$.

\section{B. Evolutions of magnetic fields}

In Fig. 1. we show the evolutions of magnetic fields induced by the slip, the anisotropic stress, and the second-order slip terms. First, we focus on the evolutions of magnetic fields before the horizon crossing. The time evolutions of magnetic fields can be approximated by a power law, and the powers of the slip and the anisotropic stress terms are $B_{\text {Slip }} \propto \eta^{1.5}$ and $B_{\text {Anis }} \propto \eta^{0.5}$, respectively. These results correspond to the previous study [63]. Furthermore, the second-order slip term is proportional to $\eta^{0.5}$ on superhorizon scales. We can explain the coincidence of the powers between the anisotropic stress and the second-order slip terms as follows. In Eq. (67), the dominant terms in early times can be estimated by using the superhorizon solutions at linear order. At first glance, the term $\delta_{\gamma}^{(1)} \times \Phi^{(1)}$ in Eq. (67) seems to give a dominant contribution to the second-order slip term. However, because both $\delta_{\gamma}^{(1)}$ and 

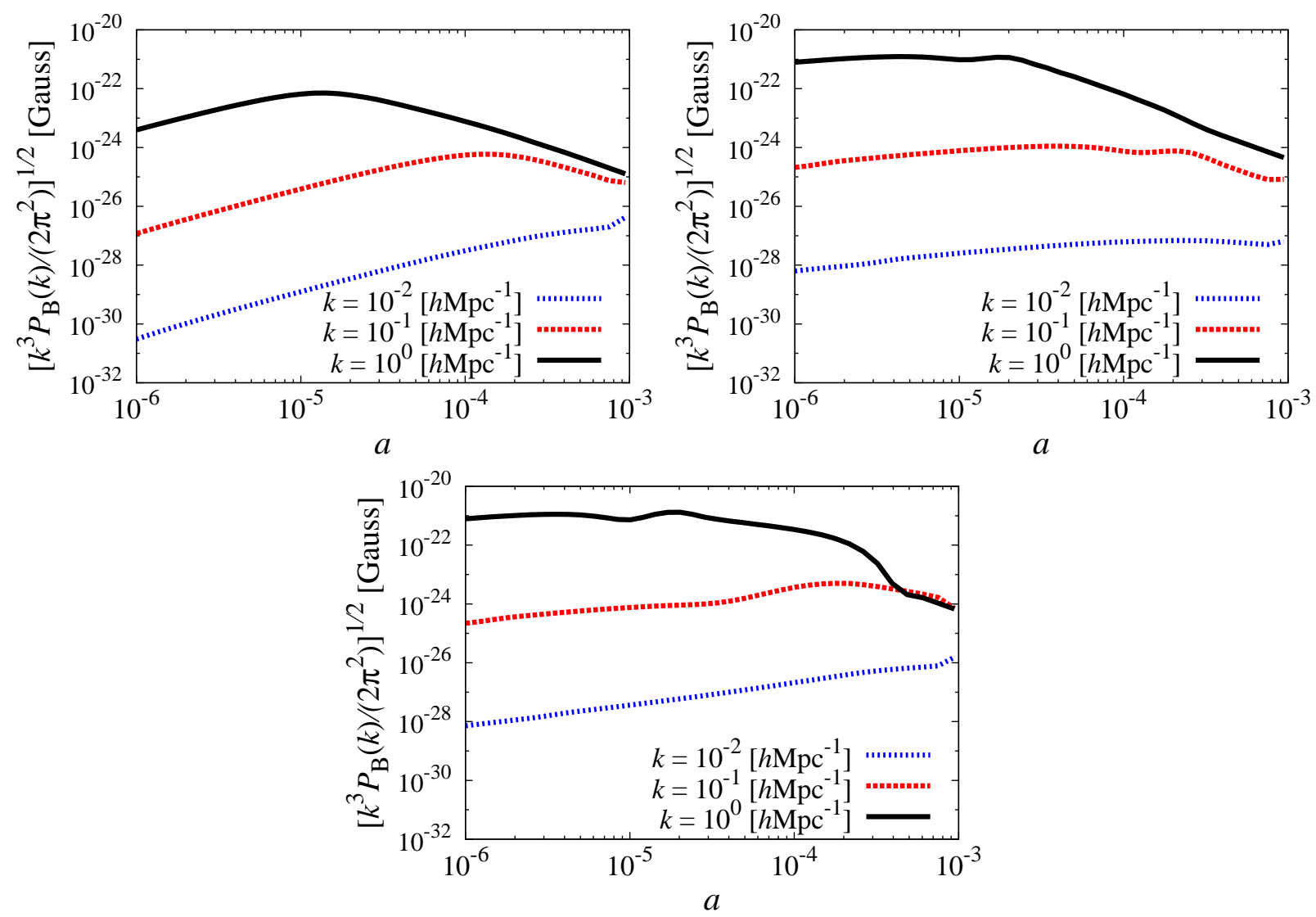

FIG. 1: Evolutions of generated magnetic fields sourced by the slip term (top left), the anisotropic stress term (top right), and the second-order slip term (bottom). We show evolutions of generated magnetic fields for wavenumbers $k=10^{-2} h \mathrm{Mpc}^{-1}$, $10^{-1} \mathrm{hMpc}^{-1}$, and $10^{0} \mathrm{hMpc}^{-1}$ as indicated in the above panels. We can see that magnetic fields at smaller scales generated earlier and their amplitudes are larger.

$\Phi^{(1)}$ are scale invariant in the Poisson gauge and according to the formula of spherical harmonics, $k_{1} \sqrt{\frac{4 \pi}{3}} Y_{1, m}^{*}\left(\hat{\boldsymbol{k}}_{1}\right)+$ $k_{2} \sqrt{\frac{4 \pi}{3}} Y_{1, m}^{*}\left(\hat{\boldsymbol{k}}_{2}\right)=k \delta_{m, 0}$, this scale invariant term vanishes in the vector mode. As a result, we find that the most dominant term in Eq. (67) is that proportional to $v_{\mathrm{b}}^{(1)} \Pi_{\gamma}^{(1)}$, which is the same form as the anisotropic stress term. Therefore, the powers of the time evolutions of the anisotropic stress and second-order slip terms coincide.

Next, we discuss the evolutions of magnetic fields after the horizon crossing. We can see that the magnetic fields from the slip and the anisotropic stress terms start to decay adiabatically as $\propto a^{-2}$, after the horizon crossing since their sources also diminish after the horizon crossing. On the other hand, magnetic fields induced by the second-order slip term do not decay adiabatically even after the horizon crossing. This arises from the fact that the second-order slip term $\delta v_{\gamma \mathrm{b}}^{(2)}$ continues to grow even after the source terms from the first-order perturbations become negligible, until the corresponding scale reaches the Silk damping scale. Therefore, the purely second-order perturbations can contribute to the magnetic field generation even if the product of the first-order perturbations is absent. When we neglect the product of the first-order perturbations, the evolution equations for magnetic fields are corresponding to the case of the first-order magnetic fields generation [39]. The relative velocity between photons and baryons, $\delta v_{\gamma \mathrm{b}}^{(2)} \lambda$ contributes to the generation of magnetic fields after the horizon crossing. This additional enhancement can be seen in the bottom of Fig. 1 at $k=10^{0} \mathrm{~h} \mathrm{Mpc}^{-1}$. However, magnetic fields induced by the additional enhancement undergo nontrivial cancellation after the Silk damping epoch and magnetic fields decay faster than the adiabatic decay that it is proportional to $a^{-2}$ [39]. The final amplitude of magnetic fields is consequently determined by the initial amplitude around horizon crossing. 

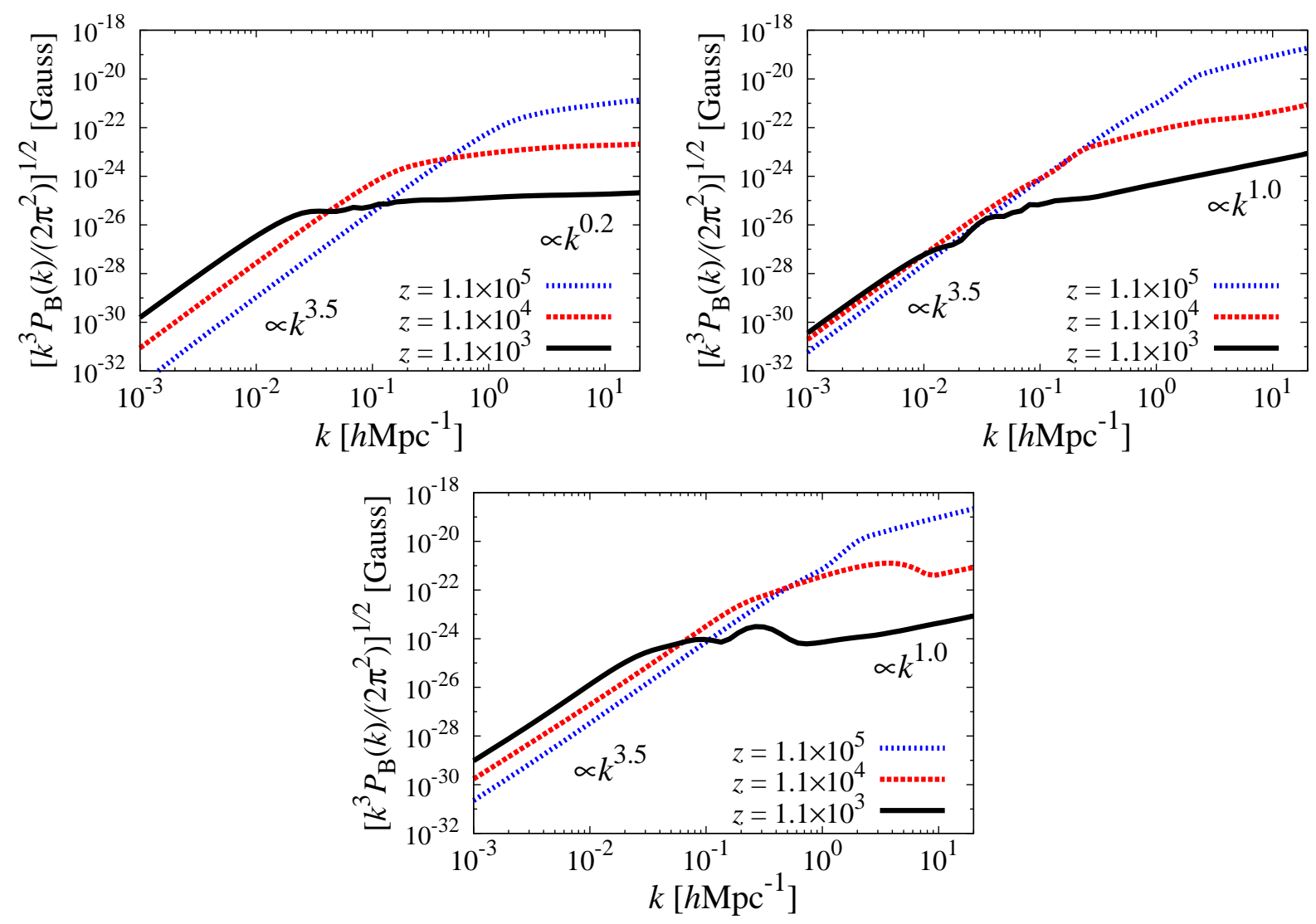

FIG. 2: Spectra of generated magnetic fields sourced by the slip term (top left), the anisotropic stress term (top right), and the second-order slip term (bottom). We show spectra of generated magnetic fields for redshifts $z=1.1 \times 10^{5}, 1.1 \times 10^{4}$, and $1.1 \times 10^{3}$ as indicated in the above panels.

\section{Spectra of magnetic fields}

Next, we show the spectra of magnetic fields induced by the slip, the anisotropic stress, and the second-order slip terms in Fig. 22 From Fig. 2, resultant magnetic fields are dominated by the anisotropic stress and second-order slip terms on smaller scales, i.e., $k \gtrsim 1.0 \mathrm{hMpc}^{-1}$, at $1+z=1100$. Conversely, on these scales, the slip term is a subdominant source for magnetic fields.

On superhorizon scales, we can see that the spectra of magnetic fields are proportional to $k^{3.5}$, which also corresponds to the results about the slip and the anisotropic stress terms in Ref. [63]. This power is also consistent with the power spectrum for causal magnetic fields [81]. From Ref. [63], the magnetic power on superhorizon scales can be estimated as below. For example, we focus on magnetic fields induced by the slip term. We can integrate the evolution equation for the second-order magnetic fields (51) and take the ensemble average as

$$
\left.\frac{k^{3}}{2 \pi^{2}} P_{B}(k)\right|_{\text {Slip }} \propto k^{5} \int \frac{d^{3} k_{1}}{(2 \pi)^{3}}\left(1-\mu_{1}^{2}\right) P_{\Phi}\left(k_{1}\right) P_{\Phi}\left(k_{2}\right)\left[S^{2}\left(k_{2}, k_{1}\right)-\frac{k_{1}}{k_{2}} S\left(k_{1}, k_{2}\right) S\left(k_{2}, k_{1}\right)\right],
$$

where $\mu_{1}=\cos \theta_{1}$ and $S\left(k_{1}, k_{2}\right)$ is defined as

$$
S\left(k_{1}, k_{2}\right)=\int d \eta a^{2}(\eta) \rho_{\gamma}^{(0)}(\eta) \delta_{\gamma}^{(1)}\left(k_{1}, \eta\right) \delta v_{\gamma \mathrm{b} 0}^{(1)}\left(k_{2}, \eta\right),
$$

where for simplicity, we omit the time dependence of $S\left(k_{1}, k_{2}\right)$. To proceed the estimation of the magnetic power, we take the limit $k / k_{1} \rightarrow 0$. This approximation can include the contributions from subhorizon scales. In this limit,

$$
k_{2}=k_{1}\left[1-\frac{k}{k_{1}} \mu_{1}+O\left(\left(k / k_{1}\right)^{2}\right)\right] \text {. }
$$


Furthermore, we can approximate the integrated source term as $S\left(k_{1}, k_{2}\right) \approx S\left(k_{2}, k_{1}\right) \approx T\left(k_{1}\right)$ since $S\left(k_{1}, k_{2}\right)$ can be treated as $k$ independent in the above limit. Then by using the fact that $P_{\Phi}(k) \propto k^{n_{s}-4}$, Eq. (80) can be rewritten as

$$
\begin{aligned}
\left.\frac{k^{3}}{2 \pi^{2}} P_{B}(k)\right|_{\text {Slip }} & \propto k^{5} \int k_{1}^{2} d k_{1} \int d \mu_{1}\left(1-\mu_{1}^{2}\right) k_{1}^{n_{s}-4} k_{2}^{n_{s}-4}\left(1-\frac{k_{1}}{k_{2}}\right) T^{2}\left(k_{1}\right) \\
& \propto k^{7} .
\end{aligned}
$$

This nonlinear power law can be seen in Fig. 2. Note that if we use the superhorizon solution only, namely, $\delta_{\gamma}^{(1)} \propto(k \eta)^{0}$ and $\delta v_{\gamma \mathrm{b} 0}^{(1)} \propto k^{3} \eta^{5}$, the magnetic power is returned as $\propto k^{8}$. This superhorizon power does not match for our numerical results.

By using our numerical code, we trace a possible cause of the discrepancies between the results in previous studies. As we noted in Sec. IVA, the transfer function of the vector mode is antisymmetric under the exchange of $k_{1}$ and $k_{2}$, and therefore, the isosceles configuration such that $k_{1}=k_{2}$ in Fourier space does not contribute in the calculation of the power spectrum. However, to achieve the result correctly, contributions from the configurations of $k_{1} \lesssim k_{2}$ and $k_{2} \lesssim k_{1}$ should be included. When these contributions are not included in the numerical calculation, the power spectrum of magnetic fields on superhorizon scales shows $\propto k^{4}$, which corresponds to the result obtained in Ref. [64].

On subhorizon scales, we can find that the spectra of magnetic fields induced by the slip, the anisotropic stress, and the second-order slip terms are proportional to $k^{0.2}, k^{1.0}$, and $k^{1.0}$, respectively. The spectra induced by the slip and the anisotropic stress terms are consistent with Ref. 63]. A noticeable feature in the spectrum induced by the second-order slip term is the additional amplification at $k \approx 5.0 \times 10^{-1} h \mathrm{Mpc}^{-1}$ at $z=1100$. As discussed in Sec. IVB this additional amplification is due to the second-order relative velocity between photons and baryons after the horizon crossing. However, this amplification is a temporary effect and the amplified magnetic fields by this effect had been erased by the epoch of Silk damping [39]. Therefore, the amplification cannot be seen for scales $k \gtrsim 1.0 \mathrm{hMpc}^{-1}$.

The above discussions and results in this subsection are valid only for the auto-power spectra of the magnetic fields from the slip, the anisotropic stress, and the second-order slip terms. In the following subsection, we focus on the total power spectrum induced by all the contributions including the cross spectra.

\section{The second-order magnetic fields}

We depict the total power spectrum at recombination in Fig. 3. It is clear that the second-order slip term gives a dominant contribution to the total magnetic fields. The amplitude of magnetic fields from the second-order slip term is 10 times larger than without the second-order slip term. However, we find that the magnetic fields from the second-order slip term are canceled out by the magnetic fields from the anisotropic stress term on small scales.

By using the tight coupling solution given by Eq. (67), this cancellation is easily understood analytically. As we mentioned in Sec. IVB, the dominant term in Eq. (67) is the anisotropic stress term given by

$$
\delta v_{\gamma \mathrm{b} \lambda}^{(2)} \simeq \frac{1}{1+R} \int \frac{d^{3} k_{1}}{(2 \pi)^{3}}\left[-\frac{5}{2} \Pi_{\gamma 0}^{(1)}\left(k_{1}\right) v_{\mathrm{b} 0}^{(1)}\left(k_{2}\right)\right] \mathcal{Y}_{1, \lambda}^{2,1}\left(\hat{\boldsymbol{k}}_{1}, \hat{\boldsymbol{k}}_{2}\right)
$$

while any other terms related to the anisotropic stress are subdominant with the baryon-photon ratio $R \propto 3 \rho_{\mathrm{b}}^{(0)} /\left(4 \rho_{\gamma}^{(0)}\right)$ as a suppression factor. By substituting this expression into Eq. (51), the evolution of magnetic fields from the secondorder slip and the anisotropic stress terms is given as

$$
\frac{d \mathcal{B}_{\lambda}}{d t} \propto\left[\frac{5}{4} \frac{1}{1+R}-\frac{5}{4}\right] v_{\mathrm{b} 0}^{(1)}\left(k_{1}\right) \Pi_{\gamma}^{(1)}\left(k_{2}\right) \mathcal{Y}_{1, \lambda}^{1,2}\left(\hat{\boldsymbol{k}}_{1}, \hat{\boldsymbol{k}}_{2}\right)
$$

where the first and the last terms in the parentheses are coming from the second-order slip term and the anisotropic stress term, respectively. We can see that the two terms in the parentheses in Eq. (86) are canceled in the radiation dominated era, where $R$ is negligibly small. However, in the matter dominated era, the baryon-photon ratio has large value and this cancellation does not occur.

The dominant contribution from the second-order slip term is canceled out by the contribution from the anisotropic stress term in the radiation dominated era. Conversely, there still remain some contributions from the second-order slip term as shown in Fig. 3 and discussed below using the tight-coupling solution. The subleading contribution from the second-order slip term can be written as

$$
\delta v_{\gamma \mathrm{b} \lambda}^{(2)} \simeq \frac{1}{1+R} \int \frac{d^{3} k_{1}}{(2 \pi)^{3}}\left[-2 \delta v_{\gamma \mathrm{b} 0}^{(1)}\left(k_{1}\right) \delta_{\gamma}^{(1)}\left(k_{2}\right)\right] \sqrt{\frac{4 \pi}{3}} Y_{1, \lambda}^{*}\left(\hat{\boldsymbol{k}}_{1}\right) .
$$




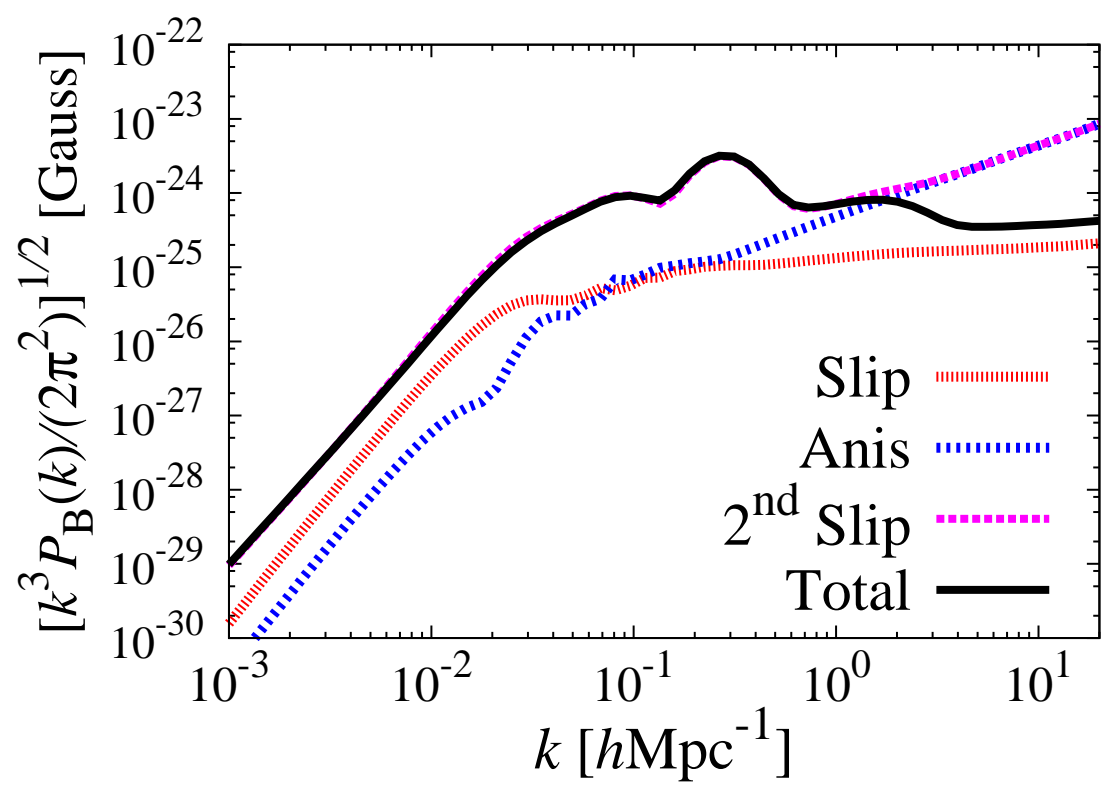

FIG. 3: Magnetic spectra generated from the slip term, the anisotropic stress term, the second-order slip term, and all terms included the cross terms at recombination $(1+z \simeq 1100)$.

Then, the evolution equation of magnetic fields induced by the slip and the second-order slip terms can be rewritten as

$$
\frac{d \mathcal{B}_{\lambda}}{d t} \propto\left[\frac{1}{1+R}+1\right] \delta v_{\gamma \mathrm{b} 0}^{(1)}\left(k_{1}\right) \delta_{\gamma}^{(1)}\left(k_{2}\right) \sqrt{\frac{4 \pi}{3}} Y_{1, \lambda}^{*}\left(\hat{\boldsymbol{k}}_{1}\right)
$$

where the first and the last terms in the parentheses are coming from the second-order slip term and the slip term, respectively. From Eq. (88), we find that the total amplitude of the spectrum of magnetic fields is twice as large as the case only with the slip term. This tendency can be seen in Fig. 3 .

Next, let us discuss the evolution of magnetic fields through the epoch of recombination. As the process of recombination proceeds, electrons form neutral hydrogen atoms with protons and the number of free electrons rapidly decreases. Accordingly the effect of the Compton scattering on generation of magnetic fields becomes negligible and magnetic fields are no longer generated through the Harrison mechanism. We show the power spectrum of the secondorder magnetic fields after recombination at $z \simeq 500$ in Fig. 4. In Fig. 4, one can find new features in the spectrum different from one at recombination on intermediate scales such as $10^{-2} h \mathrm{Mpc}^{-1} \lesssim k \lesssim 1.0 h \mathrm{Mpc}^{-1}$. These features are nearly consistent with Ref. [64]. For instance, magnetic fields induced by the slip term decrease on intermediate scales. On the other hand, magnetic fields induced by the second-order slip term are enhanced at cosmological recombination since the relative velocity is also enhanced at that era. We furthermore extend the magnetic spectrum to much smaller scales. On scales where $1.0 \mathrm{hMpc}^{-1} \gtrsim k$, magnetic fields induced by the slip, the anisotropic stress, and the second-order slip terms have the same structures as the magnetic spectrum at recombination shown in Fig. 3. As a result, the spectrum of second-order magnetic fields has a slightly blue tilt on small scales.

\section{E. Nonhelical magnetic fields}

Before closing this section, we investigate the possibility whether helical magnetic fields are generated in the Harrison mechanism or not since the helicity may play important roles in the cosmological observations [82 86]. In fact, in Ref. [87], the authors found the evidence of existence of helical magnetic fields on a few Mpc scales. It is believed that helical magnetic fields can only be generated through the process of parity violation. Because helicity is conserved in the standard magnetohydrodynamics, it is a good indicator to probe the generation mechanism of magnetic fields. We will show below that the Harrison mechanism does not induce helical magnetic fields since this mechanism relies on the standard Compton scattering which does not break the parity symmetry. 


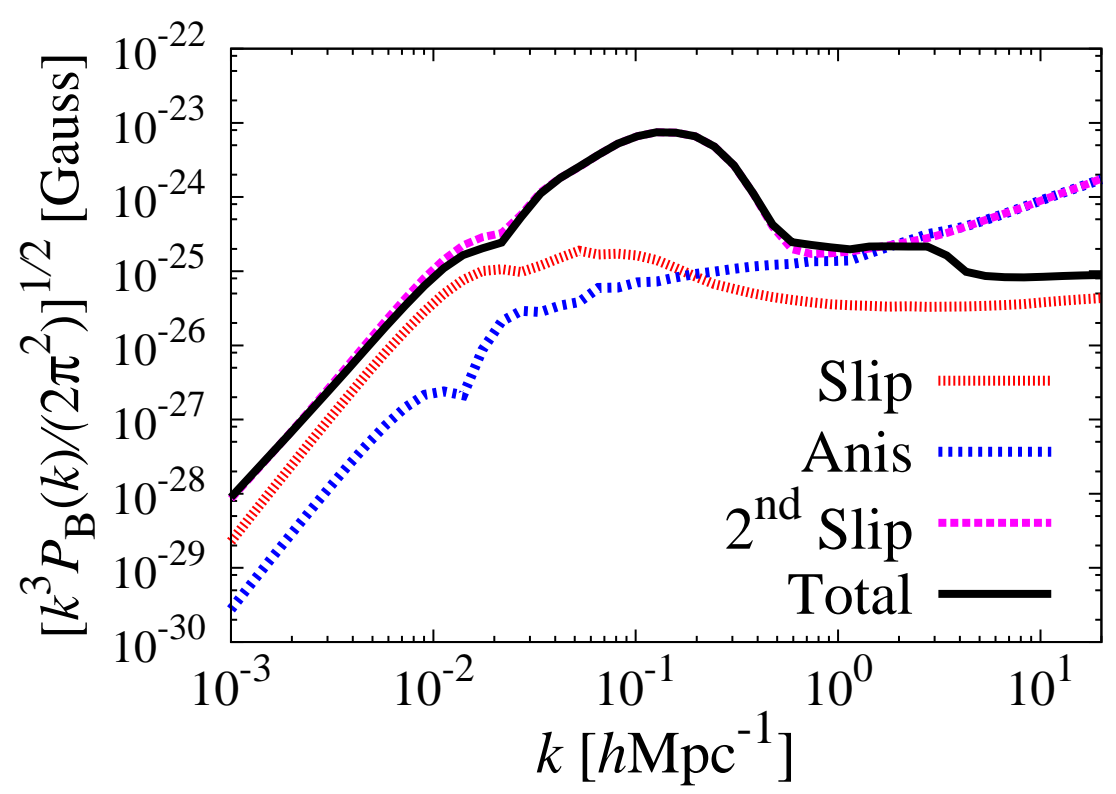

FIG. 4: Same as Fig. 3 but for after recombination as $(1+z \simeq 500)$.

At first, under the existence of helical magnetic fields, the correlation of magnetic fields can be written as

$$
\left\langle B_{i}(\boldsymbol{k}) B_{j}\left(\boldsymbol{k}^{\prime}\right)\right\rangle=(2 \pi)^{3} \delta\left(\boldsymbol{k}-\boldsymbol{k}^{\prime}\right)\left[\left(\delta_{i j}-\hat{k}_{i} \hat{k}_{j}\right) \frac{P_{B}(k)}{2}+i \epsilon_{i j k} \hat{k}^{k} \frac{P_{H}(k)}{2}\right],
$$

where $P_{B}(k)$ and $P_{H}(k)$ are the spectra of nonhelical and helical magnetic fields, respectively. The power spectrum of helical magnetic fields can be pulled by the subtraction as $\left\langle B_{i}(\boldsymbol{k}) B_{j}\left(\boldsymbol{k}^{\prime}\right)\right\rangle-\left\langle B_{j}(\boldsymbol{k}) B_{i}\left(\boldsymbol{k}^{\prime}\right)\right\rangle$. In the Harrison mechanism that is given by Eq. (45), generated magnetic fields can be symbolically expressed as

$$
B_{i}(\boldsymbol{k}) \propto \epsilon_{i a b} k^{a} \int \frac{d^{3} k_{1}}{(2 \pi)^{3}} \hat{k}_{1}^{b} f\left(\boldsymbol{k}_{1}, \boldsymbol{k}_{2}\right) X^{(1)}\left(\boldsymbol{k}_{1}\right) Y^{(1)}\left(\boldsymbol{k}_{2}\right),
$$

where $f\left(\boldsymbol{k}_{1}, \boldsymbol{k}_{2}\right)$ is an arbitrary real function of $\boldsymbol{k}_{1}$ and $\boldsymbol{k}_{2}$, and $X^{(1)}\left(\boldsymbol{k}_{1}\right)$ and $Y^{(1)}\left(\boldsymbol{k}_{2}\right)$ are the time integrals of firstorder scalar perturbations. The scalar perturbations can be decomposed into the primordial perturbation $\Phi^{(1)}(\boldsymbol{k})$ and the transfer function $X_{\mathrm{T}}\left(k_{1}\right)$ or $Y_{\mathrm{T}}\left(k_{2}\right)$ as $X^{(1)}\left(\boldsymbol{k}_{1}\right) Y^{(1)}\left(\boldsymbol{k}_{2}\right)=\Phi^{(1)}\left(\boldsymbol{k}_{1}\right) \Phi^{(1)}\left(\boldsymbol{k}_{2}\right) X_{\mathrm{T}}\left(k_{1}\right) Y_{\mathrm{T}}\left(k_{2}\right)$. Note that we use the fact that the purely second-order variables, e.g., $\delta v_{\gamma b i}$, are composed the product of the first-order scalar perturbations.

Finally, we evaluate the helical part of the power spectrum as

$$
\begin{aligned}
\left\langle B_{i}(\boldsymbol{k}) B_{j}\left(\boldsymbol{k}^{\prime}\right)\right\rangle-\left\langle B_{j}(\boldsymbol{k}) B_{i}\left(\boldsymbol{k}^{\prime}\right)\right\rangle \propto & (2 \pi)^{3} \delta\left(\boldsymbol{k}-\boldsymbol{k}^{\prime}\right)\left(\epsilon_{i a b} \epsilon_{j a^{\prime} b^{\prime}}-\epsilon_{j a b} \epsilon_{i a^{\prime} b^{\prime}}\right) k^{a} k^{a^{\prime}} \\
& \times \int \frac{d^{3} k_{1}}{(2 \pi)^{3}} f\left(\boldsymbol{k}_{1}, \boldsymbol{k}_{2}\right) X_{\mathrm{T}}\left(k_{1}\right) Y_{\mathrm{T}}\left(k_{2}\right) P_{\Phi}\left(k_{1}\right) P_{\Phi}\left(k_{2}\right) \\
& \times\left[\hat{k}_{1}^{b} \hat{k}_{1}^{b^{\prime}} f\left(\boldsymbol{k}_{1}, \boldsymbol{k}_{2}\right) X_{\mathrm{T}}\left(k_{1}\right) Y_{\mathrm{T}}\left(k_{2}\right)+\frac{1}{2}\left(\hat{k}_{1}^{b} \hat{k}_{2}^{b^{\prime}}+\hat{k}_{2}^{b} \hat{k}_{1}^{b^{\prime}}\right) f\left(\boldsymbol{k}_{2}, \boldsymbol{k}_{1}\right) X_{\mathrm{T}}\left(k_{2}\right) Y_{\mathrm{T}}\left(k_{1}\right)\right] \\
= & 0,
\end{aligned}
$$

where we symmetrize about $b \leftrightarrow b^{\prime}$ in the square bracket by using the nature of the symmetry under the exchange of $k_{1}$ and $k_{2}$. In conclusion, the Harrison mechanism cannot induce helical magnetic fields. This result is coming from the fact that general relativity and the standard Maxwell theory do not violate the parity symmetry. Therefore, the observed helical magnetic fields call for other mechanisms to explain. 


\section{CONCLUSION AND SUMMARY}

In this paper, we reinvestigate the spectrum of magnetic fields induced by cosmological perturbations through the Harrison mechanism. If we consider the cosmological perturbation theory up to the first order, the Harrison mechanism does not work since the vector mode, which is needed for this mechanism, has only a decaying solution. However, when we expand the cosmological perturbations up to the second order, the regular solution of the vector mode is excited by the first-order scalar mode. The Harrison mechanism works in the higher-order cosmological perturbation theory.

In previous studies, the spectrum of magnetic fields induced by this mechanism has been estimated. In Ref. [63], the authors show the spectrum of second-order magnetic fields induced by the product of the first-order perturbations, namely, the slip and anisotropic stress terms. Subsequently, in Ref. 64, the purely second-order slip term is included. By comparing these works, however, it is found that there are some discrepancies in the product of first-order perturbations. For example, the power law tails of the spectrum induced by the slip and anisotropic stress terms on large scales have different $k$-dependences in Refs. 63] and [64]. Furthermore, the scale-dependences of the spectrum are slightly different from each other. We find that the discrepancy can be explained by the lack of sampling in the Fourier modes at $k_{1} \approx k_{2}$ of the first-order scaler perturbations in Ref. 64, and our results agree with the ones of Ref. 63].

Let us summarize features of the magnetic fields induced by the second-order magnetic fields at cosmological recombination as follows.

- The scale dependence of magnetic fields on large scales is $\propto k^{3.5}$, which is consistent with the result in Ref. [63]. Note that magnetic fields generated by causal processes have the same power [81].

- On small scales, the spectra of magnetic fields induced by the slip, the anisotropic stress, and the second-order slip terms have the power of $k^{0.2}, k^{1.0}$, and $k^{1.0}$, respectively. In particular, the spectra of magnetic fields induced by the slip and anisotropic stress terms are consistent with the result in Ref. [63].

- The cancellation occurs between the anisotropic stress term and the second-order slip term on small scales in the tight coupling regime in the radiation dominated era, and the power of magnetic field spectrum becomes $\propto k^{0.2}$. This result indicates that the spectrum of magnetic fields cannot have the large amplitude as argued in Ref. [63].

- The spectrum of magnetic fields at cosmological recombination has a bump at $k \approx 5.0 \times 10^{-1} h \mathrm{Mpc}^{-1}$ owing to extra amplification after the horizon crossing, where the amplitude of magnetic fields is $B_{\text {rec }} \approx 5.0 \times 10^{-24}$ Gauss. However, after all, this amplification vanishes by nontrivial flipping of the relative velocity between photons and baryons discussed in Ref. [39]. This cancellation creates characteristic diffusion scales in the magnetic fields spectrum around $k \approx 10^{0} \mathrm{hMpc}^{-1}$.

- The Harrison mechanism does not work efficiency below the scale of Silk damping at the electron-positron pair creation epoch as $k_{\text {cut }} \approx 10^{9} h \mathrm{Mpc}^{-1}$, as discussed in Ref. [63]. Even if we extrapolate our numerical result toward smaller scales by using the power of $\propto k^{0.2}$ up to the cutoff scale, the amplitude of the magnetic fields at that scale cannot be larger than $10^{-23}$ Gauss, assuming that the linear density perturbations are scale invariant.

Finally, we discuss implications of the cosmological seed fields. The derived amplitude of magnetic fields at recombination has a peak about $5.0 \times 10^{-24}$ Gauss, which is sufficient for a candidate of the seed of galactic magnetic fields [17]. However, this amplitude seems to be somewhat small to explain the intergalactic magnetic fields [10, 11]. Note that, the above amplitude is derived assuming that the primordial perturbations are scale-invariant, while primordial perturbations with a blue tilt lead a larger amplitude of the magnetic fields on smaller scales.

The magnetic fields induced by the second-order perturbation must be inevitably generated in the standard cosmology, and it is possibly that the magnetic fields act as seed fields for the turbulent dynamo during the structure formation of the universe.

\section{Acknowledgments}

We thank Kenji Hasegawa for his comments to understand physical interpretation of the second-order collision term. This work was supported in part by a Grant-in-Aid for JSPS Research under Grant No. 26-63 (S.S.) and a Grant-inAid for JSPS Grant-in-Aid for Scientific Research under Grants No. 24340048 (K.I.) and 25287057 (N.S.). We also 
acknowledge the Kobayashi-Maskawa Institute for the Origin of Particles and the Universe, Nagoya University, for providing computing resources useful in conducting the research reported in this paper.

[1] L. M. Widrow, Rev.Mod.Phys. 74, 775 (2002), astro-ph/0207240.

[2] M. Giovannini, Int.J.Mod.Phys. D13, 391 (2004), astro-ph/0312614.

[3] K. Subramanian, PoS MRU, 071 (2007), 0802.2804.

[4] D. Ryu, D. R. Schleicher, R. A. Treumann, C. G. Tsagas, and L. M. Widrow, Space Sci.Rev. 166, 1 (2012), 1109.4055.

[5] A. Kandus, K. E. Kunze, and C. G. Tsagas, Phys. Rep. 505, 1 (2011), 1007.3891.

[6] K. Subramanian and J. D. Barrow, Phys.Rev.Lett. 81, 3575 (1998), astro-ph/9803261.

[7] L. M. Widrow, D. Ryu, D. R. G. Schleicher, K. Subramanian, C. G. Tsagas, and R. A. Treumann, Space Sci. Rev. 166, 37 (2012), 1109.4052.

[8] R. Durrer and A. Neronov, A\&A Rev. 21, 62 (2013), 1303.7121.

[9] A. Neronov and I. Vovk, Science 328, 73 (2010), 1006.3504.

[10] K. Takahashi, M. Mori, K. Ichiki, and S. Inoue, ApJ 744, L7 (2012), 1103.3835.

[11] K. Takahashi, M. Mori, K. Ichiki, S. Inoue, and H. Takami, ApJ 771, L42 (2013), 1303.3069.

[12] W. Chen, J. H. Buckley, and F. Ferrer (2014), 1410.7717.

[13] C. Caprini and S. Gabici (2015), 1504.00383.

[14] R. M. Kulsrud and S. W. Anderson, ApJ 396, 606 (1992).

[15] J. L. Han, R. N. Manchester, E. M. Berkhuijsen, and R. Beck, A\&A 322, 98 (1997).

[16] A. Brandenburg and K. Subramanian, Phys.Rept. 417, 1 (2005), astro-ph/0405052.

[17] A.-C. Davis, M. Lilley, and O. Tornkvist, Phys.Rev. D60, 021301 (1999), astro-ph/9904022.

[18] B. Ratra, ApJ 391, L1 (1992).

[19] K. Bamba and J. Yokoyama, Phys.Rev. D69, 043507 (2004), astro-ph/0310824.

[20] J. Martin and J. Yokoyama, JCAP 0801, 025 (2008), 0711.4307.

[21] V. Demozzi, V. Mukhanov, and H. Rubinstein, JCAP 8, 025 (2009), 0907.1030.

[22] S. Kanno, J. Soda, and M.-a. Watanabe, JCAP 12, 009 (2009), 0908.3509.

[23] T. Fujita and S. Mukohyama, JCAP 10, 034 (2012), 1205.5031.

[24] T. Fujita and S. Yokoyama, JCAP 1403, 013 (2014), 1402.0596.

[25] C. Caprini and L. Sorbo, JCAP 1410, 056 (2014), 1407.2809.

[26] S.-L. Cheng, W. Lee, and K.-W. Ng (2014), 1409.2656.

[27] T. Fujita, R. Namba, Y. Tada, N. Takeda, and H. Tashiro (2015), 1503.05802.

[28] M. Christensson, M. Hindmarsh, and A. Brandenburg, Phys.Rev. E64, 056405 (2001), astro-ph/0011321.

[29] R. Banerjee and K. Jedamzik, Phys.Rev. D70, 123003 (2004), astro-ph/0410032.

[30] M. Joyce and M. Shaposhnikov, Physical Review Letters 79, 1193 (1997), astro-ph/9703005.

[31] G. Davies and L. M. Widrow, ApJ 540, 755 (2000).

[32] H. Hanayama, K. Takahashi, K. Kotake, M. Oguri, K. Ichiki, et al., Astrophys.J. 633, 941 (2005), astro-ph/0501538.

[33] R. M. Kulsrud, R. Cen, J. P. Ostriker, and D. Ryu, Astrophys.J. 480, 481 (1997), astro-ph/9607141.

[34] Y. Fujita and T. N. Kato, Mon.Not.Roy.Astron.Soc. 364, 247 (2005), astro-ph/0508589.

[35] M. V. Medvedev, L. O. Silva, and M. Kamionkowski, Astrophys.J. 642, L1 (2006), astro-ph/0512079.

[36] E. R. Harrison, MNRAS 147, 279 (1970).

[37] K. Takahashi, K. Ichiki, H. Ohno, and H. Hanayama, Physical Review Letters 95, 121301 (2005), astro-ph/0502283.

[38] A. Lewis, Phys.Rev. D70, 043518 (2004), astro-ph/0403583.

[39] K. Ichiki, K. Takahashi, and N. Sugiyama, Phys. Rev. D 85, 043009 (2012), 1112.4705.

[40] S. Saga, M. Shiraishi, and K. Ichiki, JCAP 1410, 004 (2014), 1405.4810.

[41] L. Hollenstein, C. Caprini, R. Crittenden, and R. Maartens, Phys.Rev. D77, 063517 (2008), 0712.1667.

[42] K. Horiguchi, K. Ichiki, T. Sekiguchi, and N. Sugiyama, JCAP 1504, 007 (2015), 1501.06304.

[43] S. Saga, M. Shiraishi, K. Ichiki, and N. Sugiyama, Phys.Rev. D87, 104025 (2013), 1302.4189.

[44] W. Hu, D. Scott, and J. Silk, Phys.Rev. D49, 648 (1994), astro-ph/9305038.

[45] L. Senatore, S. Tassev, and M. Zaldarriaga, JCAP 0908, 031 (2009), 0812.3652.

[46] N. Bartolo, S. Matarrese, and A. Riotto, JCAP 0605, 010 (2006), astro-ph/0512481.

[47] N. Bartolo, S. Matarrese, and A. Riotto, JCAP 0606, 024 (2006), astro-ph/0604416.

[48] N. Bartolo, S. Matarrese, and A. Riotto, JCAP 0701, 019 (2007), astro-ph/0610110.

[49] C. Pitrou, J.-P. Uzan, and F. Bernardeau, JCAP 1007, 003 (2010), 1003.0481.

[50] C. Pitrou, Class.Quant.Grav. 26, 065006 (2009), 0809.3036.

[51] M. Beneke, C. Fidler, and K. Klingmuller, JCAP 1104, 008 (2011), 1102.1524.

[52] M. Beneke and C. Fidler, Phys.Rev. D82, 063509 (2010), 1003.1834.

[53] A. Naruko, C. Pitrou, K. Koyama, and M. Sasaki, Class.Quant.Grav. 30, 165008 (2013), 1304.6929.

[54] R. Saito, A. Naruko, T. Hiramatsu, and M. Sasaki, JCAP 1410, 051 (2014), 1409.2464.

[55] C. Fidler, K. Koyama, and G. W. Pettinari, JCAP 1504, 037 (2015), 1409.2461.

[56] T. Kobayashi, R. Maartens, T. Shiromizu, and K. Takahashi, Phys.Rev. D75, 103501 (2007), astro-ph/0701596. 
[57] K. Takahashi, K. Ichiki, and N. Sugiyama, Phys.Rev. D77, 124028 (2008), 0710.4620.

[58] S. Maeda, S. Kitagawa, T. Kobayashi, and T. Shiromizu, Class.Quant.Grav. 26, 135014 (2009), 0805.0169.

[59] R. Gopal and S. Sethi, Mon.Not.Roy.Astron.Soc. 363, 521 (2005), astro-ph/0411170.

[60] E. Nalson, A. J. Christopherson, and K. A. Malik, JCAP 1409, 023 (2014), 1312.6504.

[61] S. Matarrese, S. Mollerach, A. Notari, and A. Riotto, Phys.Rev. D71, 043502 (2005), astro-ph/0410687.

[62] K. Ichiki, K. Takahashi, H. Ohno, H. Hanayama, and N. Sugiyama, Science 311, 827 (2006), astro-ph/0603631.

[63] K. Ichiki, K. Takahashi, N. Sugiyama, H. Hanayama, and H. Ohno (2007), astro-ph/0701329.

[64] E. Fenu, C. Pitrou, and R. Maartens, MNRAS 414, 2354 (2011), 1012.2958.

[65] K. Ichiki, K. Takahashi, H. Ohno, H. Hanayama, and N. Sugiyama, Science 311, 827 (2006), astro-ph/0603631.

[66] S. Dodelson and J. M. Jubas, ApJ 439, 503 (1995), astro-ph/9308019.

[67] W. T. Hu, Ph.D. thesis, UNIVERSITY OF CALIFORNIA, BERKELEY. (1995).

[68] K. Subramanian, D. Narasimha, and S. M. Chitre, MNRAS 271, L15 (1994).

[69] K. Jedamzik, V. Katalinić, and A. V. Olinto, Phys. Rev. D 57, 3264 (1998), astro-ph/9606080.

[70] P. Ade et al. (Planck Collaboration), Astron.Astrophys. (2014), 1303.5076.

[71] P. Ade et al. (BICEP2 Collaboration), Phys.Rev.Lett. 112, 241101 (2014), 1403.3985.

[72] G. Hinshaw, D. Larson, E. Komatsu, D. N. Spergel, C. L. Bennett, J. Dunkley, M. R. Nolta, M. Halpern, R. S. Hill, N. Odegard, et al., ApJS 208, 19 (2013), 1212.5226.

[73] R. Gopal and S. K. Sethi, MNRAS 363, 521 (2005), astro-ph/0411170.

[74] M. Shiraishi, Ph.D. thesis, NAGOYA UNIVERSITY. (2012).

[75] S. Saga, K. Ichiki, and N. Sugiyama, Phys.Rev. D91, 024030 (2015), 1412.1081.

[76] K. Ichiki, K. Takahashi, and N. Sugiyama, Phys.Rev. D85, 043009 (2012), 1112.4705.

[77] C. Pitrou, Phys.Lett. B698, 1 (2011), 1012.0546.

[78] E. R. Siegel and J. N. Fry, Astrophys.J. 651, 627 (2006), astro-ph/0604526.

[79] G. W. Pettinari (2014), 1405.2280.

[80] C. L. Bennett, D. Larson, J. L. Weiland, N. Jarosik, G. Hinshaw, N. Odegard, K. M. Smith, R. S. Hill, B. Gold, M. Halpern, et al., ApJS 208, 20 (2013), 1212.5225.

[81] R. Durrer and C. Caprini, JCAP 0311, 010 (2003), astro-ph/0305059.

[82] L. Pogosian, T. Vachaspati, and S. Winitzki, Phys.Rev. D65, 083502 (2002), astro-ph/0112536.

[83] C. Caprini, R. Durrer, and T. Kahniashvili, Phys.Rev. D69, 063006 (2004), astro-ph/0304556.

[84] T. Kahniashvili and B. Ratra, Phys.Rev. D71, 103006 (2005), astro-ph/0503709.

[85] M. Shiraishi, A. Ricciardone, and S. Saga, JCAP 1311, 051 (2013), 1308.6769.

[86] M. Shiraishi, JCAP 1206, 015 (2012), 1202.2847.

[87] H. Tashiro, W. Chen, F. Ferrer, and T. Vachaspati (2013), 1310.4826. 